\title{
An assessment of the impact of local processes on dust lifting in martian climate models
}

Article

Accepted Version

Mulholland, D. P., Spiga, A., Listowski, C. and Read, P. L. (2015) An assessment of the impact of local processes on dust lifting in martian climate models. Icarus, 252. pp. 212227. ISSN 0019-1035 doi:

https://doi.org/10.1016/j.icarus.2015.01.017 Available at https://centaur.reading.ac.uk/39682/

It is advisable to refer to the publisher's version if you intend to cite from the work. See Guidance on citing.

Published version at: http://www.sciencedirect.com/science/article/pii/S0019103515000330\#

To link to this article DOI: http://dx.doi.org/10.1016/j.icarus.2015.01.017

Publisher: Elsevier

All outputs in CentAUR are protected by Intellectual Property Rights law, including copyright law. Copyright and IPR is retained by the creators or other copyright holders. Terms and conditions for use of this material are defined in the End User Agreement.

www.reading.ac.uk/centaur 
Central Archive at the University of Reading

Reading's research outputs online 


\title{
An assessment of the impact of local processes on dust lifting in Martian climate models
}

\author{
David P. Mulholland ${ }^{a}$, Aymeric Spiga ${ }^{\mathrm{b}}$, \\ Constantino Listowski ${ }^{\mathrm{c}, 1}$ and Peter L. Read ${ }^{\mathrm{d}}$

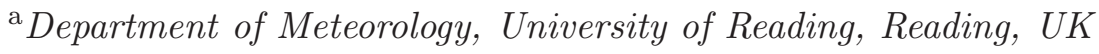 \\ ${ }^{\mathrm{b}}$ Laboratoire de Météorologie Dynamique, CNRS/UPMC/IPSL, Paris, France \\ ${ }^{\mathrm{c}}$ Université Versailles St-Quentin; Sorbonne Universités, UPMC Univ. Paris 06; \\ CNRS/INSU, LATMOS-IPSL, Guyancourt, France \\ dAtmospheric, Oceanic \& Planetary Physics, University of Oxford, Oxford, UK \\ Copyright (C) 2014 David P. Mulholland, Aymeric Spiga, Constantino Listowski and Peter L. Read
}

Number of pages: 55

Number of figures: 13

$\overline{1 \text { Now }}$ at British Antarctic Survey, Cambridge, UK 
Proposed Running Head:

Local processes in Martian dust lifting

Please send Editorial Correspondence to:

David P. Mulholland

Department of Meteorology, University of Reading,

Earley Gate, PO Box 243, Reading RG6 6BB, UK

Email: d.p.mulholland@reading.ac.uk

Phone: +44 (0)118 3787762 


\section{ABSTRACT}

Simulation of the lifting of dust from the planetary surface is of substantially greater importance on Mars than on Earth, due to the fundamental role that atmospheric dust plays in the former's climate, yet the dust emission param-

5 eterisations used to date in Martian global climate models (MGCMs) lag, understandably, behind their terrestrial counterparts in terms of sophistication. Recent developments in estimating surface roughness length over all Martian terrains and in modelling atmospheric circulations at regional to local scales (less than $\mathrm{O}(100 \mathrm{~km})$ ) presents an opportunity to formulate an improved wind stress lifting parameterisation. We have upgraded the conventional scheme by including the spatially varying roughness length in the lifting parameterisation in a fully consistent manner (thereby correcting a possible underestimation of the true threshold level for wind stress lifting), and used a modification to account for deviations from neutral stability in the surface layer. Following these improvements, it is found that wind speeds at typical MGCM resolution never reach the lifting threshold at most gridpoints — winds fall particularly short in the southern midlatitudes, where mean roughness is large. Sub-grid scale variability, manifested in both the near-surface wind field and the surface roughness, is then considered, and is found to be a crucial means of bridging the gap between model winds and thresholds. Both forms of small-scale variability contribute to the formation of dust emission 'hotspots': areas within the model gridbox with particularly favourable conditions for lifting, namely a smooth surface combined with strong near-surface gusts. Such small-scale emission could in fact be particularly influential on Mars, due both to the intense positive radiative feedbacks that can drive storm growth and a strong hysteresis effect on saltation. By modelling this variability, dust lifting is pre- 
dicted at the locations at which dust storms are frequently observed, including the flushing storm sources of Chryse and Utopia, and southern midlatitude areas from which larger storms tend to initiate, such as Hellas and Solis Planum. so The seasonal cycle of emission, which includes a double-peaked structure in northern autumn and winter, also appears realistic. Significant increases to lifting rates are produced for any sensible choices of parameters controlling the sub-grid distributions used, but results are sensitive to the smallest scale of variability considered, which high-resolution modelling suggests should be $35 \mathrm{O}(1 \mathrm{~km})$ or less. Use of such models in future will permit the use of a diagnosed (rather than prescribed) variable gustiness intensity, which should further enhance dust lifting in the southern hemisphere in particular.

Keywords: Aeolian processes; Mars, atmosphere; Mars, surface; Terrestrial planets 
Atmospheric dust on Mars is of fundamental importance to the overall climate system, as it can alter — through strong absorption of incoming solar radiation - the strength of the global-scale circulation [Haberle et al., 1982] and the characteristics of atmospheric eddies [Hourdin et al., 1995], as well as act as condensation nuclei for water or $\mathrm{CO}_{2}$ ice clouds [Määttänen et al., 2005]. Martian atmospheric dust exhibits strong spatial and temporal variability in optical depth. A spectacular example of this variability is the occurence of planet-encircling dust storms in southern summer [Zurek and Martin, 1993]. Those events are, however, relatively rare, and the majority of Martian years involve dust lifting, emission, and transport in a more regionally confined fashion [e.g., Smith, 2004]. Regional dust storms have been observed to occur with greater regularity in the midlatitudes of both hemispheres [Cantor et al., 2001], although dust storms occur in equatorial and tropical regions too, albeit perhaps more sporadically [Määttänen et al., 2009]. Interestingly,

the depth of coverage of dust on the planetary surface has also been found to be rather inhomogeneous, implying a long-term removal of dust from southern midlatitudes and deposition in northern subtropics [Ruff and Christensen, 2002].

A key question in understanding the dust cycle on Mars, as well as on Earth and on Titan, is how dust particles are lifted from the surface into the atmosphere [Kok et al., 2012, for a review]. On Mars, the lifting of dust from the surface appears as a strongly threshold-dependent process, with a positive radiative feedback that amplifies the interannual variability of the Martian climate, through the initiation of dust storms of varying sizes. This fact was made 
apparent through the use of interactive dust lifting schemes in Martian global climate models (MGCMs) [Murphy et al., 1995, Newman et al., 2002a]. Two distinct lifting mechanisms have been parameterised in MGCMs: near-surface wind stress [Bagnold, 1954], and dust devils [dusty turbulent whirlwinds, see Balme and Greeley, 2006, for a review]. MGCM and observational studies suggest that wind stress lifting, rather than dust devils, plays the dominant role in the initiation of regional and global dust storms [Newman et al., 2002b, Kahre et al., 2006, Montabone et al., 2005, Cantor et al., 2006], making this mechanism the more important to MGCMs attempting to replicate the observed degree of interannual climate variability.

The knowledge of physical processes and feedbacks responsible for dust lifting on Mars is still in relative infancy. Several putative key phenomena have not been considered thus far, due to a lack of either relevant observational constraints or appropriate modelling tools.

(1) The aerodynamic surface roughness length, $z_{0}$, controls the transfer of momentum between the surface and the surface layer, i.e. the lowermost part of the Planetary Boundary Layer (PBL). It is thus key to the wind stress lifting mechanism; yet a globally uniform value of $z_{0}$ has, to date, been used in all existing MGCMs. Furthermore, dust lifting thresholds include a dependence on $z_{0}$ that has been neglected in MGCMs so far. Recent global mapping of $z_{0}$ at high resolution by Hébrard et al. [2012] provides an opportunity to assess the influence of $z_{0}$ spatial variability on surface layer physics and lifting thresholds.

(2) Discussions in the literature about dust lifting on Mars (and subsequent lifting parameterisations in MGCMs) do not take into account the effect of varying atmospheric stability on the vertical profile of wind in the 
surface layer [Monin and Obukhov, 1954, Garratt, 1992]. This directly affects the conversion of atmospheric momentum into surface drag. Since the Martian PBL undergoes a strong diurnal cycle between daytime superadiabatic and nighttime ultra-stable regimes [e.g., Schofield et al., 1997, Smith et al., 2006], this stability effect should be included when modelling dust lifting.

(3) MGCM horizontal grid spacing is typically a few hundred kilometres, which is inadequate for representing important mesoscale circulations, such as slope-induced flows [Tyler et al., 2002, Rafkin and Michaels, 2003, Spiga et al., 2011] and turbulent PBL circulations resolved by Large-Eddy Simulations (LES) [Michaels and Rafkin, 2004, Spiga et al., 2010]. The former is referred to hereafter as 'mesoscale variability', the latter as 'turbulent gustiness', and both of them as 'sub-grid scale (wind speed) variability' (from an MGCM-grid perspective). As a result, unless a specific parameterisation of those effects is included [e.g., Michaels, 2006], an MGCM will severely underestimate the maximum horizontal wind speed reached close to the surface, and therefore the potential for various lifting mechanisms to be activated. Indeed, it has been found that dust loading in the African desert is more highly correlated with near-surface gustiness than it is with the mean wind speed at GCM resolution [Engelstaedter and Washington, 2007]. In addition, sub-grid scale variability in roughness length $z_{0}$ should be accounted for, since small variations in $z_{0}$ can strongly affect dust lifting thresholds [e.g., Raupach et al., 1993].

Surface roughness length, atmospheric stability, and sub-grid scale variability 115 could act together to modify both the wind stress exerted by the atmosphere on the surface, and the threshold wind stress for dust particles to be lifted 
from the surface. For instance, the 'hotspot' theory of dust lifting on Earth [Gillette, 1999] implies that appropriate parameterisations of sub-grid scale variability are needed to simulate the observed dust lifting rates [Cakmur et al., 2004, Okin, 2005]. In Martian studies, apart from dust devil parameterisations [Rennó et al., 1998] which account for only a part of the dust lifting related to turbulent gustiness [another one being horizontal winds associated with convective cells, see e.g., Spiga and Lewis, 2010, Figure 5], none of the three aforementioned phenomena has been examined nor taken into account in global-scale dust lifting models, aside from an early attempt by Newman et al. [2002b] to explore the use of parameterised turbulent gustiness. It is the aim of this paper to explore, in the Martian environment, the nature and magnitude of each of these three components, as well as their impact both on dust lifting rates, and the spatial and seasonal variability of dust lifting events.

\section{Dust lifting thresholds at MGCM resolution}

In this section, three issues, each important in the calculation of dust lifting rates at the scale of an MGCM horizontal grid, are dealt with in turn: spatial heterogeneity in surface roughness length (including a presentation of a global map of this variable), calculation of the lifting threshold itself using formulae derived from physical principles, and the impact of atmospheric stability in the conversion of atmospheric wind to drag velocity. The first and third of these are new additions to the existing studies of Martian dust lifting with GCMs. 
For many years, the only measure of the aerodynamic roughness length, $z_{0}$, at the Martian surface came from the Viking landers, for which a value in the range $0.1-1 \mathrm{~cm}$ was estimated [Sutton et al., 1978]. As a result, values within this range have been assumed in MGCMs thus far, without spatial variation. Other surface landers have made similar estimates of $z_{0}$, including $3 \mathrm{~cm}$ at the Mars Pathfinder landing site [Sullivan et al., 2000], and a range of values $0.07-0.60 \mathrm{~cm}$ along the path of the Spirit Rover [Greeley et al., 2008]. The latter indicates though that significant variations in roughness length within short distances are possible over the planet's surface. On Earth, it has been found that accounting for spatial heterogeneity in surface characteristics greatly improves the performance of dust emission models [e.g., Callot et al., 2000].

Recently, Hébrard et al. [2012] yielded a global $z_{0}$ map covering the full Martian surface, at a resolution of $\frac{1}{8}^{\circ} \times \frac{1}{8}^{\circ}$, well in excess of typical MGCM resolutions ( $\sim 5^{\circ}$ in latitude and longitude). The dataset was derived from rock abundance maps obtained from TES thermal inertia data [Nowicki and Christensen, 2007]. The completed $z_{0}$ map is displayed in Fig. 1. At full resolution, values of $z_{0}$ span three orders of magnitude, from $\sim 0.001 \mathrm{~cm}$ to $2 \mathrm{~cm}$ (a comparable range of values to the range encountered on Earth [MacKinnon et al., 2004]). The southern hemisphere, particularly in the $30-60^{\circ} \mathrm{S}$ region that is key for dust storm activity in southern summer, is characterised by large roughness lengths $(\gtrsim 0.5 \mathrm{~cm})$. By contrast, the vast majority of the northern hemisphere is smooth, with values of $z_{0} \lesssim 0.1 \mathrm{~cm}$ over much of the low-thermal-inertia plains at $15^{\circ} \mathrm{S}-30^{\circ} \mathrm{N}$. The flushing dust storm channels of Acidalia-Chryse 


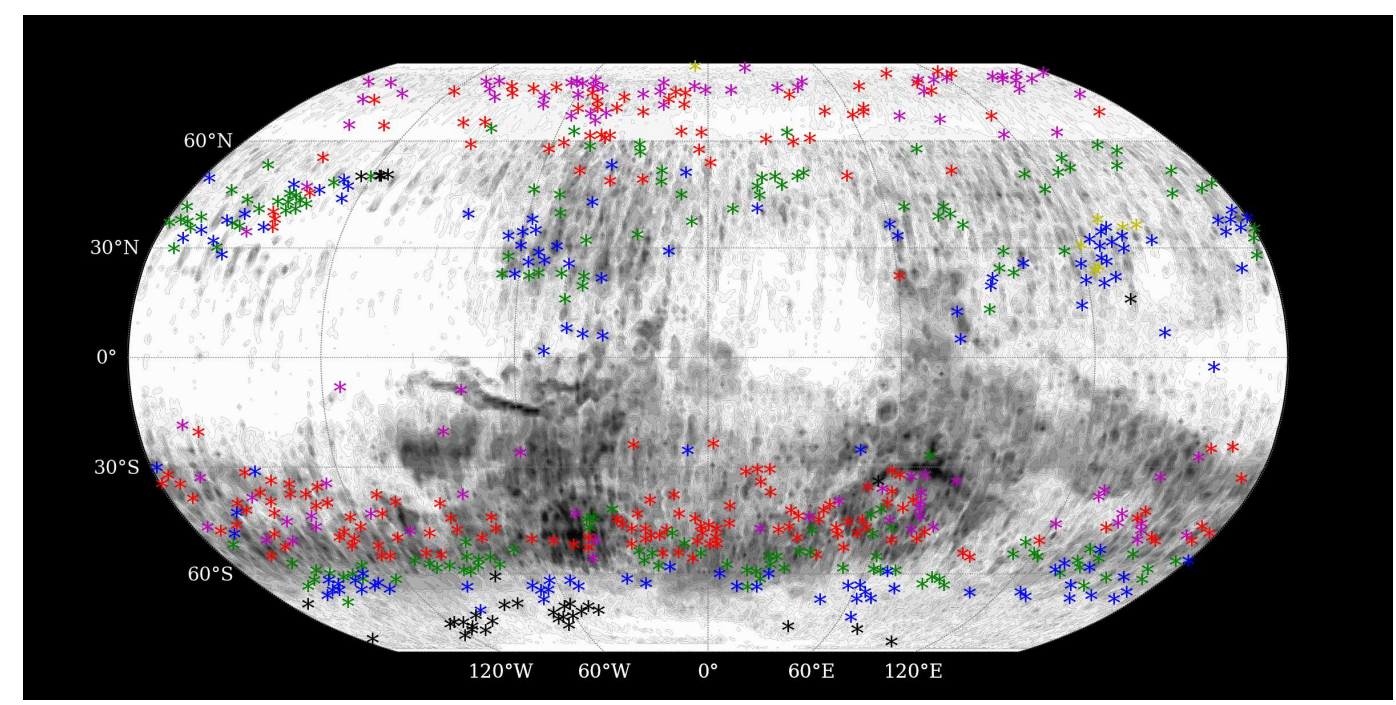

Fig. 1. Global map of surface roughness length (shading), from the data described in Hébrard et al. [2012]. The range covers approximately $0.001 \mathrm{~cm}$ (white) to $2 \mathrm{~cm}$ (black), and shading is on a logarithmic scale. Stars mark the location of dust storms observed by Cantor et al. [2001], coloured according to the time of year at which they occurred, from $L_{s}=109-129^{\circ}$ (yellow) through purple, red, green and blue to 250-274 (black). The map features streaks in the meridional direction, an artefact of the tracks followed by the satellite from which the data were retrieved.

$\left(\sim 60^{\circ} \mathrm{W}\right)$ and Utopia-Isidis $\left(\sim 90^{\circ} \mathrm{E}\right)$, however, exhibit fairly large roughness lengths. This Hébrard et al. global dataset features a significant number of pixels with $z_{0}$ close to $1 \mathrm{~cm}$, confirming that this value was a reasonable choice for use in MGCMs in previous work, but the $z_{0}$ field is very inhomogeneous, and $84 \%$ of the surface possesses a roughness length below $1 \mathrm{~cm}$. The largest value in the map is $2.02 \mathrm{~cm}$. A large inhomogeneity was also found by Heavens et al. [2008], who previously produced two different versions of the $z_{0}$ map (but which appear less consistent with in situ measurements than the Hébrard map does [see Hébrard et al., 2012]).

Overlaid in Fig. 1 are the locations at which dust storms were observed by 
Cantor et al. [2001] over a substantial fraction of one Martian year. Although the detection of a dust storm does not strictly imply local dust lifting, the majority of the storms marked were smaller than regional in extent, such that it may be assumed that the initial lifting activity occurred relatively close (in global terms) to the observed storm site. The majority of these small dust storms occurred along the seasonally shifting edges of the polar $\mathrm{CO}_{2}$ ice caps, where large surface temperature gradients lead to conditions of high baroclinic instability (as well as a 'sea breeze' circulation [Toigo et al., 2002]), and therefore to strong peak wind speeds. Note that dust lifting apparently occurs at locations with a wide range of $z_{0}$ values, from the smooth north polar regions to the rougher southern midlatitudes. Dust storms seem to be absent from a band at $20-30^{\circ} \mathrm{S}$, which features some of the largest $z_{0}$ values, though this may be primarily due to the fact that the seasonal $\mathrm{CO}_{2}$ cap (and therefore the winter baroclinic zone) never extends this far north. Nevertheless, a robust outcome from Fig. 1 appears to be that in regions of $z_{0} \gtrsim 0.5 \mathrm{~cm}$, dust storms are frequent occurrences.

\subsection{Effect of $z_{0}$ on the lifting threshold $u_{*}^{t}$}

On Earth, after near-surface wind speed, the roughness length $z_{0}$ is thought to be the most important control on dust lifting rates [Gillette, 1999]. The rate of dust emission for a given drag velocity depends on how much of this drag is exerted on the erodible (dust-covered) surface, and how much is 'lost' on the roughness elements — rocks and pebbles that impede the flow and shelter parts of the surface. Dust emission therefore occurs with greater amplitude, or at a lower threshold drag velocity, where the surface roughness length is small, 
while emission may be extremely rare in locations with large $z_{0}$ [Laurent et al., 2008]. On Mars, however, relatively large roughness lengths do not seem to prohibit dust lifting, as evidenced by the array of dust storms marked in Fig. 1. An interesting point of additional complexity is that some dust is likely to rest on top of the non-erodible elements [Jakosky et al., 2006], after settling out of the atmosphere, and could be more easily lifted due its exposure to the unimpeded flow just above the roughness elements. This is beyond the scope of this paper, and we simply assume that all dust lies on the surface, distributed homogeneously amongst the non-erodibles.

Wind stress dust lifting is usually treated in MGCMs as a threshold-dependent process, occurring only when the drag velocity $u_{*}$ exceeds a threshold value $u_{*}^{t}$ [e.g., Iversen and White, 1982], i.e. when

$$
u_{*}>u_{*}^{t}
$$

When the wind is strong enough, erodible material becomes mobilised and flows along the soil bed. Conventionally, this is assumed to occur most readily for sand-sized particles, of diameter $\sim 100 \mu \mathrm{m}$, in which case the process is called saltation. These particles are too heavy to be maintained in suspension, but can eject smaller particles (whose stronger interparticle cohesion prevents them from being lifted directly by the wind) into the atmosphere through their impacts on the surface ('sandblasting'). Therefore, the resulting vertical dust flux is generally considered to be proportional to this saltation flux [e.g., Marticorena and Bergametti, 1995]. The combination of saltation and sandblasting is believed to be the primary mechanism for non-convective dust emission on Earth, and possibly on Mars [Greeley and Iversen, 1985]. There has been a suggestion [Sullivan et al., 2008] that direct detachment of 
loosely bound, low-density clumps of dust particles is in fact the preferred lifting mechanism on Mars — we address this possibility in section 2.3. In any case, the essential point is that non-convective dust lifting is a strongly threshold-dependent process, and this threshold is dependent on roughness length.

The threshold drag velocity on a surface covered by non-erodible elements is obtained as a modification to the threshold that applies to a smooth surface (free of obstacles), $u_{* s}^{t}$, as

$$
u_{*}^{t}=\frac{u_{* s}^{t}}{f_{\mathrm{eff}}\left(z_{0}\right)},
$$

225 where $f_{\text {eff }}\left(z_{0}\right)$ is the drag partition function, which accounts for the distribution of momentum between the underlying surface and roughness elements, and is a function of $z_{0}$. In previous modelling studies on Mars, although a uniform $z_{0}$ value of $\sim 1 \mathrm{~cm}$ has been used in the calculation of $u_{*}$ (see later), the effect of $z_{0}$ on the threshold drag velocity has been neglected: $f_{\text {eff }}$ has been implicitly set equal to one (equivalent to assuming $z_{0}=0$ ), making lifting thresholds at all model gridpoints quite significantly lower than they are theorised to be in reality. In fact, $f_{\text {eff }}$ is always less than one, making the threshold velocity for a rough surface larger than that for a smooth surface, particle size and local conditions being the same. This key problem has been overlooked in previous studies that attempted to use a physically based threshold [Newman et al., 2002a, Kahre et al., 2006], which as a result used thresholds that were unrealistically low over much of the planet.

Many different formulations for the drag partition coefficient $f_{\text {eff }}$ have been proposed in the terrestrial literature [Raupach et al., 1993, Alfaro and Gomes, 1995, Marticorena and Bergametti, 1995, MacKinnon et al., 2004]: these are 


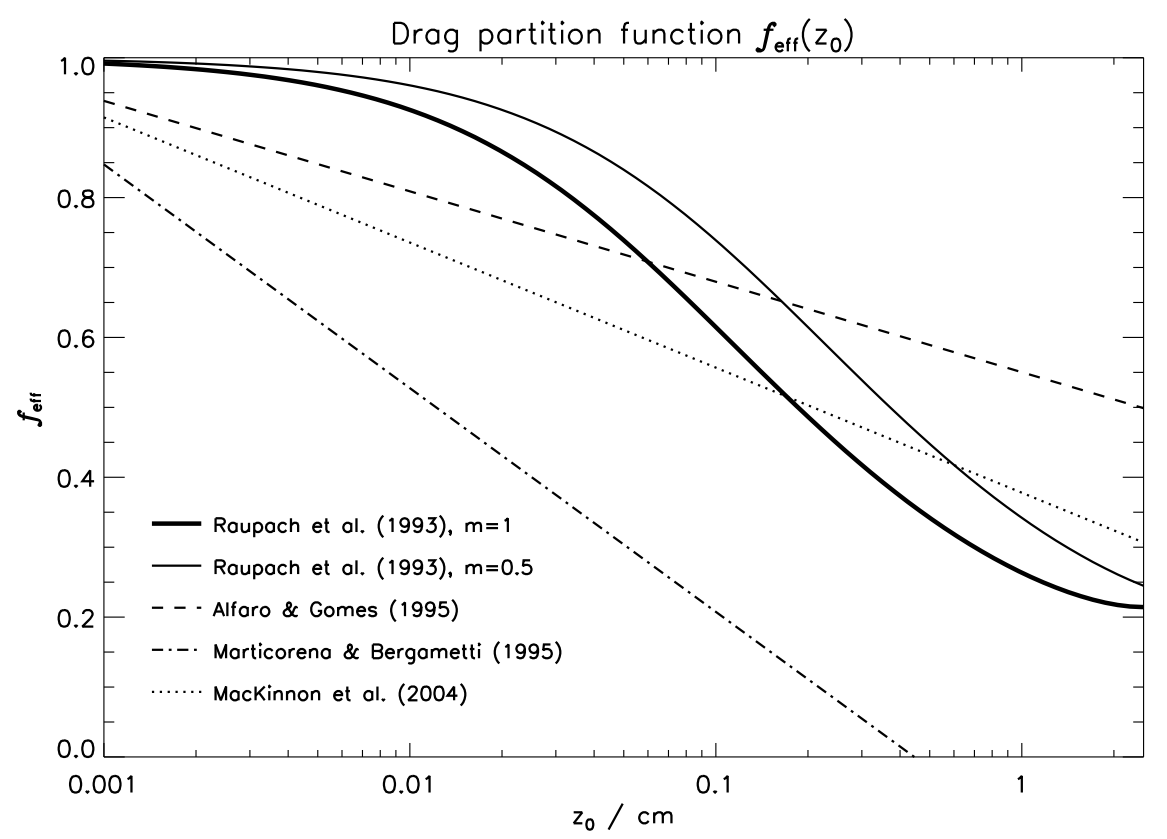

Fig. 2. A comparison of various forms for $f_{\text {eff }}$ proposed in the literature. The form of Raupach et al. [1993], an extension of which was presented by Okin [2005], is expressed as a function of roughness density, $\lambda$, rather than roughness length $z_{0}$, so a conversion from $\lambda$ to $z_{0}$ is needed. For simplicity, we use $z_{0}=\frac{H \lambda}{2}$ from Wooding et al. [1973], where $H$ is the average rock height, which we set to $H=20 \mathrm{~cm}$, in preference to a more sophisticated semi-empirical relationship derived from formulae in Hébrard et al. [2012]. The thick solid line, from Raupach et al. [1993] with $m=1$, is the function that we adopt for the majority of this paper.

plotted, for comparison, in Fig. 2. Which one is suitable for Mars? All the forms of $f_{\text {eff }}$ approach unity as $z_{0}$ approaches the value that applies to a 'smooth' surface, and each function predicts that $f_{\text {eff }}$ is less than $\sim 0.6$ at roughness lengths of $0.5 \mathrm{~cm}$ and above. Yet there are (potentially important) differences between the various forms.

The Marticorena and Bergametti [1995] function relies on Arya's [1975] model which assumes a logarithmic wind profile downwind of an obstacle (roughness 
element) and matches it with the background (also assumed) logarithmic profile in order to get $u_{*}^{t}$ as a function of $u_{* s}^{t}$, the threshold for the smooth surface between roughness elements. The model is validated by wind-tunnel profiles of Marshall [1971], which used cylinders and hemispherical obstacles as the roughness elements. We decide to rule out the Marticorena and Bergametti function for Mars, as it does not allow any lifting above $z_{0}=0.4 \mathrm{~cm}$ and requires very strong winds at $z_{0}$ above $\sim 0.1 \mathrm{~cm}$, which appears to be 255 at odds with the observed dust storm occurrences shown in Fig. 1. It might be explained by the fact that Arya's model was first designed to represent situations where roughness elements are not too closely spaced [Marticorena and Bergametti, 1995], with roughness density $\lambda<0.05$, or roughness length $z_{0} \lesssim 0.5 \mathrm{~cm}$, which is exceeded at a considerable number of Martian sites.

The MacKinnon et al. [2004] function is a modified form of Marticorena and Bergametti's, allowing for larger roughness lengths, though this fit appears to involve an unphysically large value for one of the parameters in the equation: 'roughness factor' $x$ (see their Eq. (20)), which is supposed to be of the order of the distance between two obstacles [Arya, 1975], but for which a value of $\mathrm{O}\left(10^{4}\right)$ is used. MacKinnon et al. [2004] were aiming to match field measurements where roughness elements comprised vegetation (plants, grass, etc.), which is thus a different scenario to Marshall's [1971] wind-tunnel experiments, and to the Martian surface, where roughness elements (rocks) are less porous. In addition to the shortcomings of Marticorena and Bergametti 270 [1995] and MacKinnon et al. [2004] with respect to our purpose for Mars, the initial Arya [1975] model on which both functions rely was built assuming logarithmic wind profiles, which is an assumption that should be discarded in Martian conditions (see section 2.4). 
Another function, from Alfaro and Gomes [1995], is a fit to a single set of experimental wind-tunnel data, by studying the influence of the roughness length of the underlying surface $\left(z_{0, s}\right)$, and not of the non-erodible elements $\left(z_{0}\right)$. They produced an updated expression for $f_{\text {eff }}$, which also relies on Arya's [1975] model, by closely taking into account the possible variations of $z_{0, s}$ with different surface textures. However, because their function was derived from a restricted set of experimental data we will rather concentrate on what appears to be the most reliable expression for $f_{\text {eff }}$ so far: that of Raupach et al. [1993].

The Raupach et al. formulation, which used the Marshall [1971] data in its derivation, has since been tested against a number of wind tunnel and field experiments [Wolfe and Nickling, 1996, Gillies et al., 2007]. Okin [2008] updated the Raupach formulation, evaluating it against a wider range of experimental data, and added a correction to the high- $z_{0}$ end at which the original Raupach fit lost validity. Okin did not provide a readily applicable equation for $f_{\text {eff }}$, but his retention of the basic Raupach functional shape, its support from abundant measurement studies, and the considerations exposed above regarding the other functions, lead us to favour the Raupach et al. [1993] approach as the most reliable of the functions presented here, at least for the Martian case. Hence, in subsequent sections, we primarily use the Raupach et al. drag partition function, but comparative calculations using the MacKinnon et al. [2004] form (which gives a smaller variation in $f_{\text {eff }}$ along the relevant range of $z_{0}$ ) are also described, to illustrate the sensitivity to this choice. Using the conversion $z_{0}=\frac{H \lambda}{2}$, where $H=20 \mathrm{~cm}$ [Wooding et al., 1973], the Raupach et al. function is

$$
f_{\mathrm{eff}}=\sqrt{\frac{1}{\left(1-\frac{2 m \sigma z_{0}}{H}\right)\left(1+\frac{2 m \beta z_{0}}{H}\right)}}
$$


where $(\beta, \sigma, m)=(170,2,1)$. The three parameters represent the ratio of the drag coefficients of an isolated non-erodible element to that of the smooth surface, the ratio of basal to frontal area of the non-erodible elements, and the ratio of average to peak surface stress over a bare surface. In the absence of any Mars-specific information, we use the set of values tentatively recommended by Raupach et al. [1993]; we note support in the model results of Okin [2008], however, for a steeper drop-off of $f_{\text {eff }}$ at high $z_{0}$ when non-porous obstacles (i.e. Martian rocks) are involved, which suggests that the use of the larger $m=1$ is appropriate, as seen in Fig. 2.

The use of a realistic drag partition function $f_{\text {eff }}$, which has values significantly smaller than 1 at high $z_{0}$, results in substantial increases to the calculated lifting thresholds $u_{*}^{t}$ for medium-to-large roughness lengths. However, the impact of $z_{0}$ on model dust lifting is more complicated, since $z_{0}$ also enters into the calculation of the drag velocity $u_{*}$ from the atmospheric wind speed $(u)$ near the ground. For conditions in which a logarithmic vertical wind profile can be assumed [Priestley, 1959],

$$
u_{*}=\frac{\kappa u\left(z_{1}\right)}{\ln \left(\frac{z_{1}}{z_{0}}\right)}
$$

where $\kappa$ is von Kármán's constant $(=0.4)$, and $z_{1}$ is height of the lowest model level, usually a few metres in MGCMs (this applies to boundary layer conditions of neutral stability: we discuss the more general case in section 2.4). From equation (2), the larger $z_{0}$ is, the larger $u_{*}$; therefore, rough surfaces feel a stronger drag force from a given near-surface wind than smooth surfaces do. The effect of surface roughness on lifting at a given $u\left(z_{1}\right)$ is dependent on the balance between the increase to $u_{*}$ and the increase to $u_{*}^{t}$ with increasing $z_{0}$. This balance is sensitive to the particular expression chosen for $f_{\text {eff }}$. Combining (1) and (2), we can speak of a threshold wind speed at height $z_{1}$, that 


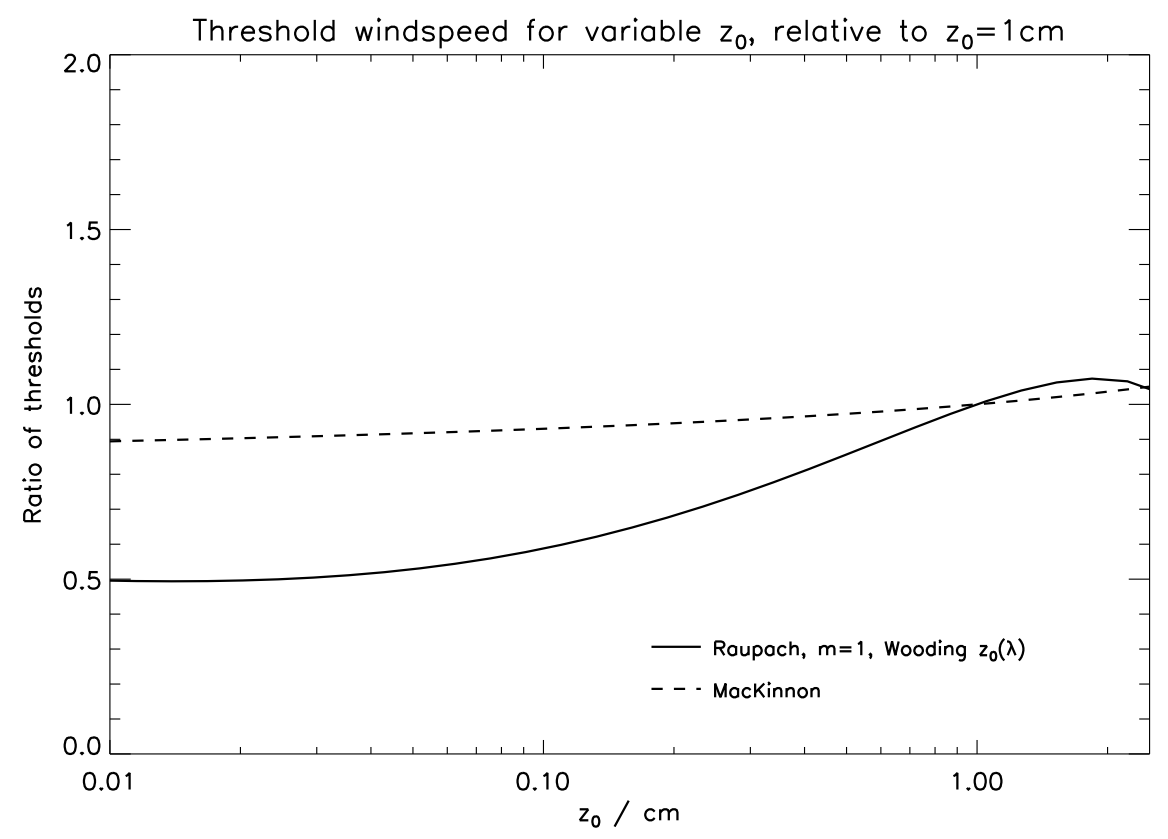

Fig. 3. $u\left(z_{1}\right)^{t} / u\left(z_{1}\right)_{z_{0}=1 \mathrm{~cm}}^{t}$, the ratio between threshold wind speeds (at height $z_{1}$ ) using a variable $z_{0}$ and using a fixed $z_{0}$ of $1 \mathrm{~cm}$, for the Raupach (+Wooding) and MacKinnon drag partition functions, under conditions of neutral stability at the near-surface. Deviations of the lines from a ratio of one imply that model dust lifting rates will vary as a function of $z_{0}$. The Raupach function is known to be unreliable beyond $\sim 2 \mathrm{~cm}$ (seen in the turning of the solid curve at the upper end of the plotted range), but the largest roughness length in the full-resolution Martian map is $2.02 \mathrm{~cm}$, and $99.8 \%$ of pixels have values below $1.8 \mathrm{~cm}$; at $5^{\circ}$ resolution, as used in section 2.5 , the largest value is $1.46 \mathrm{~cm}$.

corresponds to a drag velocity $u_{*}^{t}$ :

$$
u\left(z_{1}\right)^{t}=\frac{u_{* s}^{t} \ln \left(\frac{z_{1}}{z_{0}}\right)}{\kappa f_{\text {eff }}\left(z_{0}\right) .}
$$

It is this quantity, therefore, that controls the viability of model dust lifting at a given $u\left(z_{1}\right)$ (note, also, that $\left.u_{*} / u_{*}^{t}=u\left(z_{1}\right) / u\left(z_{1}\right)^{t}\right)$.

Fig. 3 shows the ratio between $u\left(z_{1}\right)^{t}$ and $u\left(z_{1}\right)_{z_{0}=1 \mathrm{~cm}}^{t}$, the threshold wind 
speed when a fixed $z_{0}$ of $1 \mathrm{~cm}$ is used, as a function of $z_{0}$, for conditions 295 of neutral stability. For the Raupach et al. [1993] formula (solid line), $f_{\text {eff }}$ decreases rapidly as $z_{0}$ becomes large, and as a result, $u_{*}^{t}$ increases more rapidly than $u_{*}$ does, meaning that a larger wind speed is required at high $z_{0}$ than at lower $z_{0}$ values. The difference in threshold wind speed over the range of $z_{0}$ encountered on Mars is around a factor of two. For the MacKinnon et al. [2004] formula (dashed line), on the other hand, lifting is only slightly more difficult at large $z_{0}$ than at small $z_{0}$ — wind speeds vary by only around $10 \%$ over the relevant $z_{0}$ range.

This dependence is important for two reasons: firstly, it will affect the spatial distribution of annual mean dust lifting in an MGCM (the Raupach form will make lifting from the high- $z_{0}$ areas of the southern hemisphere relatively unfavourable, in comparison to the MacKinnon form); secondly, it will determine the extent to which sub-grid scale variability in $z_{0}$ is important for MGCM dust lifting (since the dependence applies down to scales much smaller than an MGCM gridbox). Sub-grid scale variability, through the contribution of hotspots to total gridbox lifted flux, will be most important when threshold $u_{*}^{t}$ varies most strongly with $z_{0}$, while variability in $z_{0}$ will be unimportant if $u_{*}^{t}$ is in fact insensitive to $z_{0}$. Since we are treating the Raupach et al. [1993] partition function as the more plausible of the two, the lesson of Fig. 3 is that dust lifting should be relatively difficult in the Martian southern hemisphere, and that sub-grid scale variation in $z_{0}$ will be important in this regard. 


\subsection{Explicit calculation of $u_{*}^{t}$}

Equation (1) shows that, in addition to $z_{0}$ (through $f_{\text {eff }}$ ), the threshold drag velocity on a smooth surface, $u_{* s}^{t}$, is needed to determine the threshold for lifting on a rough surface. It can be obtained from one of several available theoretical or semi-empirical formulae. A formulation that has been often used in dust lifting studies on both Earth and Mars [e.g., Kang et al., 2011, Kok et al., 2012, Menut et al., 2013, Mulholland et al., 2013] is that of Shao and $\mathrm{Lu}[2000]$ :

$$
u_{* s}^{t}=\sqrt{A_{N}\left(\sigma_{p} g D_{p}+\frac{\gamma}{\rho D_{p}}\right)},
$$

where $\sigma_{p}=\frac{\rho_{d}}{\rho}$, the ratio of dust particle density to atmospheric density, $D_{p}$ is the dust particle diameter, $g$ is the gravitational acceleration, $\gamma \approx$ $3 \times 10^{-4} \mathrm{~kg} \mathrm{~s}^{-2}$ and $A_{N} \approx 0.0123$. The constants $\gamma$ and $A_{N}$ encompass particle arrangement, particle Reynolds number at threshold drag velocity and the interparticle electrostatic force, and their values, which are not Earthspecific, were determined empirically by fitting to wind tunnel data. This formula can be differentiated with respect to particle diameter to find the minimum threshold velocity:

$$
u_{* s}^{t}=\sqrt{2 A_{N}}\left(\frac{\gamma \rho_{d} g}{\rho^{2}}\right)^{\frac{1}{4}},
$$

which applies to the most 'erodible' (most easily mobilisable) particles, of diameter $D_{p}=\sqrt{\frac{\gamma}{\rho_{d} g}}$.

With surface roughness included, combining (1) and (4), the threshold drag velocity is then given by:

$$
u_{*}^{t}=\frac{\sqrt{2 A_{N}}\left(\frac{\gamma \rho_{d} g}{\rho^{2}}\right)^{\frac{1}{4}}}{f_{\mathrm{eff}}} .
$$


The value of this threshold depends crucially on the dust particle density $\rho_{d}$, and in this respect there is considerable uncertainty over which is most appropriate for the Martian surface. Conventionally, dust lifting modelling studies have used a 'bulk' density of $\rho_{d}=2500 \mathrm{~kg} \mathrm{~m}^{-3}$ or similar for Martian dust, which leads to a minimum threshold for sand-sized particles, with diameter around $180 \mu \mathrm{m}$. It is therefore assumed that saltation of these sand grains causes the raising of micron-sized grains which remain in suspension, in agreement with size measurements of atmospheric dust [e.g., Wolff et al., 2006]. With a near-surface atmospheric density of $\rho=0.02 \mathrm{~kg} \mathrm{~m}^{-3}$, (4) then gives a smooth threshold velocity of $u_{* s}^{t}=1.4 \mathrm{~ms}^{-1}$. Other calculations of sizedependent $u_{* s}^{t}$ have been employed, most notably that of Greeley and Iversen [1985], but if the assumption that lifting begins at the lowest point of the $u_{* s}^{t}\left(D_{p}\right)$ curve is retained, the resulting value does not differ greatly from (4) [Park and Park, 2010]. The uncertainty in the threshold, then, is apparently fairly small, at least within the conventional saltation-emission theory for wind stress dust lifting.

However, it has been noted by numerous authors [e.g., Zimbelman, 2000, Sullivan et al., 2008] that evidence of saltating sand particles is rarely found on Mars, yet the injection of micron-sized particles into the atmosphere is evidently ubiquitous. A suggested explanation for this apparent paradox is that micron-sized dust grains clump together on the surface, possibly aided by electrification, to form loosely packed dust aggregates [Merrison et al., 2004]. These structures are much more erodible than sand particles, due to their lower density, and Martian winds may frequently be strong enough to lift the aggregates directly from the surface [Merrison et al., 2007]. Once in suspension, the tensile strength of these aggregates has been suggested to be 
extremely low, leading to their rapid break-up into constituent, micron-sized grains [Sullivan et al., 2008].

Possible values for the density and diameter of surface dust aggregates are uncertain, but using an aggregate density of $\rho_{d(a g g)}=750 \mathrm{~kg} \mathrm{~m}^{-3}$ and diameter $D_{p}=500 \mu \mathrm{m}$ (from Sullivan et al. [2009]), equation (3) produces a threshold of around $80 \%$ of that for individual sand grains, while plausible variation in density and diameter could reduce this further, to around $70 \%$ of the sand particle threshold $\left(u_{*_{s}}^{t} \sim 1.0 \mathrm{~m} \mathrm{~s}^{-1}\right.$, equivalent to a surface stress of $\sim 0.02 \mathrm{~Pa}$, in agreement with Merrison et al. [2007]).

Since part of the focus in this work is to develop the representation of dust 355 lifting in Martian regional and global models, we retain the default assumption of modellers to date, that saltation of sand grains is responsible for the emission of micron-sized particles. However, we note that this model of Martian dust lifting appears increasingly to be in question (at least in some regions where a lack of sand movement has been observed) and so we keep in mind the possible reduction of $u_{*}^{t}$ that would be justified if direct detachment of lowdensity aggregates is in fact the dominant process.

\subsection{Consideration of non-neutral atmospheric stability for $u_{*}$}

Lifting rates are also dependent, for a particular near-surface wind speed, on atmospheric stability in the PBL. Under conditions of neutral stability, drag velocity $u_{*}$ is given by (2), but in non-neutral conditions the assumption of a logarithmic wind profile does not hold, and a more complicated form of $u_{*}$ is required. A thermal plume model, which includes a surface flux parame- 
terisation that uses Monin-Ubukhov similarity theory to account for variable near-surface stability, was recently added to the LMD MGCM by Colaitis et al. 370 [2013], where it is described in detail. The key result of the parameterisation for our purpose is that the expression for $u_{*}$ in (2) is multiplied by $\sqrt{f_{m}(R i)}$, the stability function for momentum, which varies with the bulk Richardson number $R i$ in the lowest model layer. This function takes different forms depending on the atmospheric stability, resulting in a decrease in $u_{*}$ (compared 375 to the neutral case) when the boundary layer is stable $(R i>0$, commonly experienced at night), and an increase in $u_{*}$ in unstable conditions $(R i<0$, which typically applies from mid-morning to late afternoon). $u_{*}$ can be modified further by a parameterisation of turbulent gustiness that is included in the calculation of vertical fluxes of heat and momentum in the boundary layer.

380 The impact on $u_{*}$ is believed to be fairly minor, but enhanced upward momentum fluxes can reduce daytime $u_{*}$, in opposition to increases resulting from instability.

The changes to $u_{*}$ are usually relatively small — of the order of $20 \%$ or less [see the plots of $u_{*}^{2}$ in Colaitis et al., 2013, Figure 6] — and peak daytime drag velocities can be either increased or reduced, depending on ambient conditions. The precise nature of these changes have not yet been investigated. Nevertheless, since the Colaïtis et al. scheme is included in the Mars Climate Database used for calculations in the following subsection, we include the variable stability method as part of the 'baseline' dust lifting model, constructed throughout this section, that is evaluated below using gridbox-mean wind speed and $z_{0}$ fields. Compared to a neutral stability approach, this new surface scheme is expected to have only a small net effect on the frequency of dust lifting events, but could slightly alter their spatiotemporal distribution. 


\subsection{MGCM drag velocities: comparison of $u_{*}$ to threshold $u_{*}^{t}$} mance of this dust lifting model under Martian conditions. The Mars Climate Database (MCD) version 5.0 [Millour et al., 2012] contains output from the LMD MGCM, from simulations performed using a variety of different forcing (dust opacity, solar intensity) fields. Simulations were performed at $u_{*}$

The peak value of $u_{*} / u_{*}^{t}$ reached at each gridpoint when using gridbox-mean values for wind speed and roughness length is plotted in Fig. 4. Values greater than one are reached at various points in the northern high latitudes, and at a single point in the southern hemisphere, within the Hellas basin on the northeastern slope, but over most of the southern extratropics the annual peak $u_{*}$ is less than $40 \%$ of the local threshold. Note that some of the largest drag 


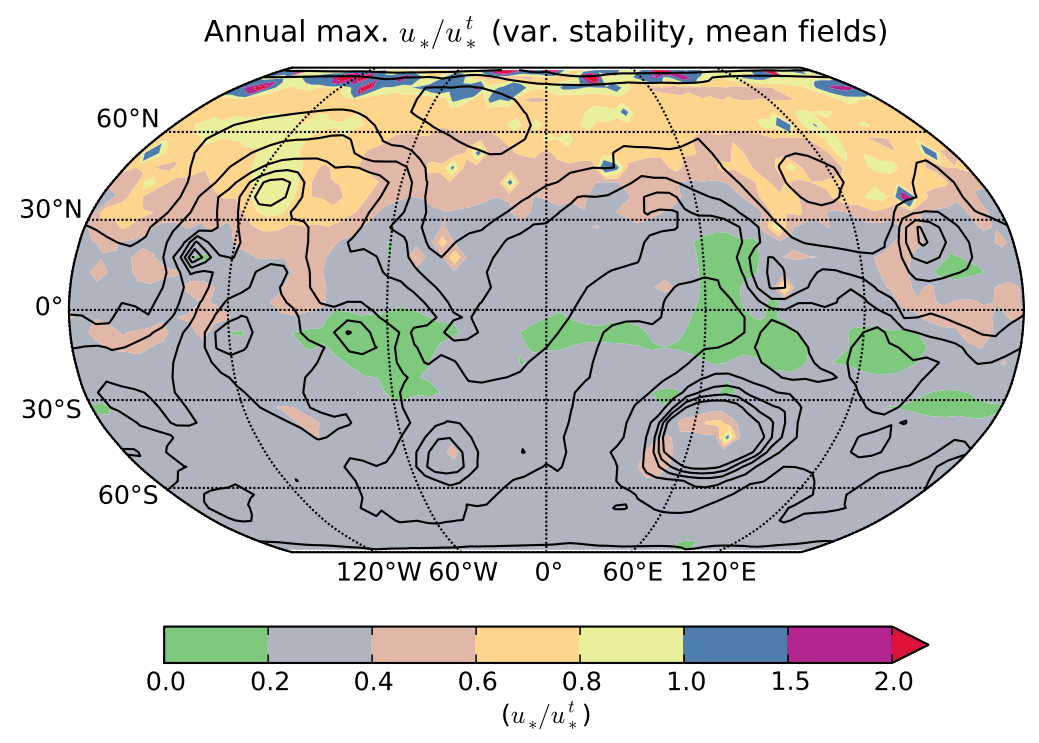

Fig. 4. The peak drag velocity reached, as a fraction of the local threshold $u_{*}^{t}$, during the 'Climatological' MCD year, using GCM mean fields for $z_{0}$ and $u\left(z_{1}\right)$ (with $z_{1}=5 \mathrm{~m}$ ), and the variable stability scheme for converting to $u_{*}$. The surface topography is drawn in black contours, as it is in several subsequent figures.

velocities actually occur in the southern subtropics (in spring), but thresholds are so large in this region that they are never even approached, with the exception of the small area in Hellas. A similar result is obtained using the MacKinnon et al. [2004] drag partition function, but with somewhat increased values in the southern midlatitudes.

Since the majority of the excursions above the local threshold at $70-90^{\circ} \mathrm{N}$ occur during periods of $\mathrm{CO}_{2}$ ice surface cover, when it is conventionally assumed that no dust lifting is possible (it is also worth noting that the uncertainty on $z_{0}$ values in the Hébrard et al. [2012] map is largest in the high latitudes), 
lifting events are shown here to be extremely rare in the LMD MGCM - and virtually non-existent in the southern hemisphere - which is very much at frequent occurrences, particularly in the midlatitudes [Cantor et al., 2001]. If the lower thresholds that are applicable to direct lifting of low-density aggregates are applied instead, values in the plot should be scaled by a factor of $\sim 1.4$ - this would mean the activation of lifting at further gridpoints in where lifting would remain extremely limited.

The large differences between peak MGCM drag velocities and the threshold drag velocities calculated using the true surface roughness point to a crucial role for sub-grid scale variability in dust lifting. As we explore in the next section, parameterisation of unresolved variability may be required both for near-surface winds and for the surface roughness field, and could therefore impact both $u_{*}$ and $u_{*}^{t}$. As well as altering the ability, in a global sense, of the MGCM to initiate dust lifting events, parameterising sub-grid scale variability could alter the spatial distribution of the most favourable sites for lifting potentially reducing the northern hemispheric dominance seen in these MCD gridbox-mean calculations.

\section{$3 \quad$ Exploring sub-grid scale variability}

We now evaluate the extent to which unresolved spatial variations in surface roughness and in horizontal wind strength can be sources of additional lifted dust flux in an MGCM. In both cases, the hotspot theory [Gillette, 1999] predicts that the majority of dust lifting will be concentrated in areas smaller than 
the typical MGCM horizontal resolution, and that - for gridbox-mean wind speeds below the mean lifting threshold — total lifting will be underestimated at coarse resolution as a result. We estimate the changes in the effective $u_{*}^{t}$ and $u_{*}$ that result from these effects, and in the next section discuss whether or not this then brings thresholds within the reach of peak MGCM drag velocities. In particular we use the ratio $u_{*} / u_{*}^{t}$ in several of the following plots, since this is the crucial quantity for dust lifting with variable $z_{0}$.

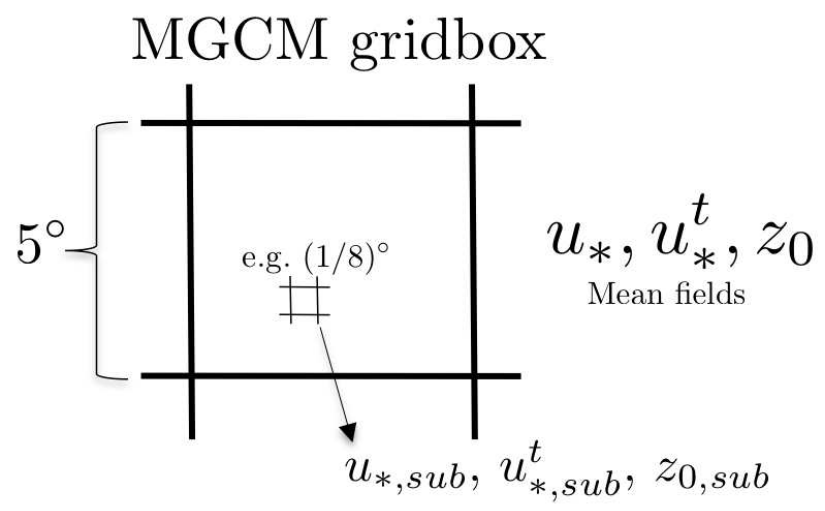

Fig. 5. Schematic representation of variable conditions within an MGCM gridbox, and the notation used to distinguish between gridbox-mean and sub-grid variables.

To clearly distinguish between gridbox-mean and higher-resolution variables, we introduce variables $u_{* \text {,sub }}, u_{* \text { sub }}^{t}$ and $z_{0, \text { sub }}$ to represent respectively drag velocity, threshold drag velocity and surface roughness length values within a $5^{\circ}$ MGCM gridbox, while retaining $u_{*}, u_{*}^{t}$ and $z_{0}$ as the gridbox-mean equivalents. The situation is illustrated by Fig. 5 . 


\subsection{Surface roughness length variability: $z_{0, \text { sub }}<z_{0}$}

465

Although individual pixels in the surface roughness length map shown in Fig. 1 carry with them (in some cases, substantial) uncertainties, the availability of a map at a spatial resolution significantly higher than that typically used by MGCMs provides an opportunity to assess the impact on predicted dust lifting rates of sub-grid scale variability in roughness length. As shown in Fig. 3 , for a given MGCM wind speed, lifting will be favoured at locations within a gridbox with small $z_{0}$; that is, points at which $z_{0, \text { sub }} \ll z_{0}$. The activation point for lifting (i.e. the lowest wind speed at which some lifting occurs) is controlled by the site within a gridbox with the largest ratio of drag velocity to threshold drag velocity, $\left(u_{*, \mathrm{sub}} / u_{*, \mathrm{sub}}^{t}\right)_{\max }$. Note that this quantity may be 475 greater than one even if the $5^{\circ}$-mean equivalent, $u_{*} / u_{*}^{t}$, is less than one, in which case the dust lifting rate will be systematically underestimated if $z_{0}$ variations are not accounted for.

Fig. 6 shows, for the Raupach drag partition function, the ratio of these two quantities, that is $\frac{\left(u_{*, \mathrm{sub}} / u_{*, \mathrm{sub}}^{t}\right)_{\max }}{u_{*} / u_{*}^{t}}$ (which is independent of wind speed). Where this ratio is $>1$, dust lifting will occur at a lower wind speed when sub-grid scale $z_{0}$ variability is parameterised than it does using gridbox-mean values. To avoid possible undue influence from single outlying pixels in the $\frac{1}{8}^{\circ}$ map, synthetic sub-grid values $z_{0 \text {,sub }}$ were generated using standard deviations calculated for each $5^{\circ}$ gridbox from the high-resolution map, assuming that 485 $z_{0, \text { sub }}$ are distributed log-normally within $5^{\circ}$ gridboxes (Supplementary Information, Fig. S2 shows that this assumption is reasonable). The results shown here are for a sub-grid resolution of $\sim \frac{1}{6}^{\circ}$, and are not greatly different to the result of simply using the $\frac{1}{8}^{\circ}$ map directly. 


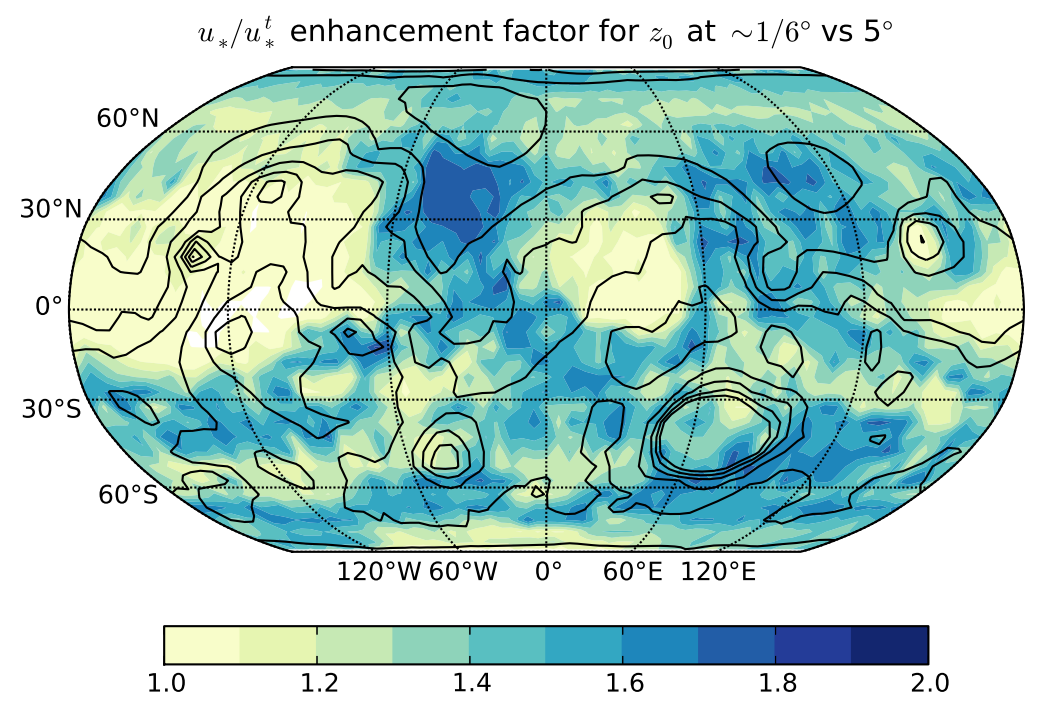

Fig. 6. The ratio $\frac{\left(u_{*, \mathrm{sub}} / u_{*, \mathrm{sub}}^{t}\right)_{\max }}{u_{*} / u_{*}^{t}}$ at a sub-grid resolution of $\sim \frac{1}{6}^{\circ}$, for a homogeneous mean $\left(5^{\circ}\right)$ near-surface wind speed, using the Raupach et al. [1993] drag partition function, accounting for sub-grid scale $z_{0}$ variability by generating $z_{0, \text { sub }}$ distributions with standard deviations $\sigma_{g}\left(z_{0}\right)$ to match those calculated from the full-resolution Hébrard et al. [2012] map.

The sub-grid quantity $\left(u_{* \text {,sub }} / u_{* \text {,sub }}^{t}\right)_{\text {max }}$ is twice as large as its gridbox-mean counterpart in southern midlatitude regions, implying that dust lifting will begin at a drag velocity equal to half of the mean threshold $u_{*}^{t}$ (or equivalently, at a wind speed of half the mean threshold wind speed $\left.u\left(z_{1}\right)^{t}\right)$. The effect of $z_{0}$ variability varies significantly with location: in the smooth northern hemisphere plains, the ratio of drag velocity to threshold drag velocity is only $\sim 10 \%$ larger with sub-grid variables than with mean variables. The spatial distribution shows that the enhancement factor increases more rapidly with increasing $z_{0}$ than it does with increasing standard deviation of $z_{0}$ values within the $5^{\circ}$ gridbox, $z_{0}$ and $\sigma_{g}\left(z_{0}\right)$ being strongly spatially anti-correlated 
(Supplementary Information, Fig. S2). This situation exists due to the dependence of threshold on $1 / f_{\text {eff }}$ seen in Fig. 3 : in a $5^{\circ}$ box with a low $z_{0}$ value, the threshold drag velocity is already close to $u_{* s}^{t}$, but the threshold for a $z_{0, \text { sub }} \sim 0.1 \mathrm{~cm}$ point within a box with $z_{0} \sim 1 \mathrm{~cm}$ is significantly lower than the $5^{\circ}$ threshold. In other words, a large spread of $z_{0, \text { sub }}$ values creates subgrid points lying further to the left of the mean along the (Raupach) curve of Fig. 3, but a large mean $z_{0}$ is required for this displacement to make lifting significantly easier. So, it is especially important to account for sub-grid scale $z_{0}$ variation in southern midlatitudes, where the box-mean $z_{0}$ is large, despite the relatively low $z_{0}$ spreads found here.

The values plotted in this figure represent the multiplicative factor that may be applied to Fig. 4 to account for sub-grid scale variability in $z_{0}$, at $\sim \frac{1}{6}^{\circ}$ resolution. From comparison of the two figures, it can therefore be seen that $z_{0}$ variability could increase the annual peak $u_{*} / u_{*}^{t}$ significantly in many regions, and in the southern hemisphere this could be sufficient to push model $u_{*}$ above the threshold at several more gridpoints in Hellas, and perhaps at several other isolated points. The increase to peak $u_{*} / u_{*}^{t}$ will be even greater as the $z_{0}$ resolution is increased further, particularly in regions of large mean $z_{0}$, where there is greater scope for sub-grid areas to exhibit significantly larger $f_{\text {eff }}$ values than the gridbox mean.

A quite different situation arises when using the MacKinnon form of $f_{\text {eff }}$ (see Supplementary Information, Fig. S3), due to its weaker dependence on $z_{0}$ : enhancements to $u_{*} / u_{*}^{t}$ of no more than $10 \%$ are produced, meaning that subgrid $z_{0}$ variations are largely irrelevant to model dust lifting. Also, the fairly uniform gradient of the dashed line in Fig. 3 means that variations in $z_{0}$ affect $u_{*} / u_{*}^{t}$ independently of the mean $z_{0}$ value, such that enhancements to $u_{*} / u_{*}^{t}$ 
inantly in the northern hemisphere. The magnitude of the impact on dust lifting rates of sub-grid scale $z_{0}$ variability is, therefore, strongly dependent on the drag partition function used.

\subsection{Wind speed variability $: u_{*, \mathrm{sub}}>u_{*}$}

The other important source of variability unresolved by an MGCM lies in wind speed itself. Within a GCM gridbox of order $100 \mathrm{~km}$, there will exist smaller areas in which the surface wind speed is significantly stronger than the gridbox mean value: $\left(u\left(z_{1}\right)_{\operatorname{sub}}\right)_{\max }>u\left(z_{1}\right)$, and, if $z_{0}$ variability is set aside, $\left(u_{*, \mathrm{sub}}\right)_{\max }>u_{*}$. At horizontal scales of around 1-10 km, mesoscale circulations 535 (fronts, slope winds) could impact winds at regional scales and trigger dust lifting: this variability is resolved by limited-area mesoscale models and highresolution MGCMs [e.g., Tyler et al., 2002]. Yet there is another crucial source of wind variability that is left unresolved by most MGCMs and mesoscale models, and deserves further attention: turbulent gustiness. when a certain value is predicted by an MGCM for the 'ambient' wind speed, what is the typical maximum wind speed encountered within an interval of about half an hour, if one were to take into account turbulent gustiness? This also relates to the above-mentioned hotspot theory, while highlighting the important point that favoured lifting sites may be transient in nature, and shift location within a gridbox in response to a changing wind field. To address the question, we analyse results from the LMD LES model [Spiga et al., 2010], run with a background wind speed equivalent to a drag velocity 


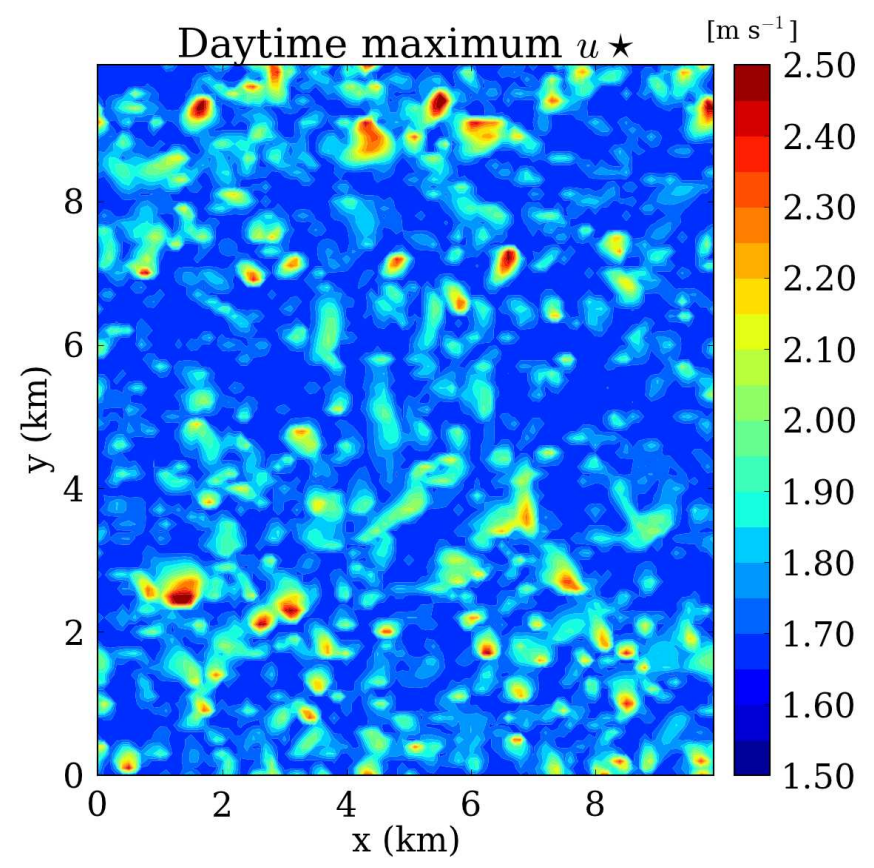

Fig. 7. Daily maximum drag velocity from an LMD LES model simulation, at horizontal resolution $100 \mathrm{~m}$, in which the background $u_{*}$ was $1 \mathrm{~m} \mathrm{~s}^{-1}$.

$u_{*}=1 \mathrm{~m} \mathrm{~s}^{-1}$ (CaseA.w30 in Colaïtis et al. [2013]), with a horizontal resolution 550 of $100 \mathrm{~m}$ and a domain size of $10 \mathrm{~km} \times 10 \mathrm{~km}$.

Fig. 7 reveals that in many parts of the domain, the daily maximum drag velocity exceeded twice the ambient drag velocity (i.e. $\left(u_{*, \text { sub }}\right)_{\max } \geq 2 u_{*}$ ); furthermore, every gridpoint produced a daily maximum of at least $1.5 u_{*}$. That is, at every gridpoint, over a full day of model integration, $u_{*}$ varied by at least $50 \%$ about the mean value. In an MGCM, the unresolved temporal variability covers much shorter periods (timesteps of around 30 minutes), so the underestimation of $u_{*}$ peaks will be less severe. However, information on a second form of variability, spatial, can be obtained from Fig. 7. The domain-mean daytime maximum $u_{*}$ lies below $80 \%$ of the domain-maximum value of $\sim 2.5 \mathrm{~m} \mathrm{~s}^{-1}$, which indicates the level of spatial wind variability that 
can be expected from the division of a domain into 10,000 sub-grid points. For an MGCM gridbox, which at $5^{\circ}$ resolution has a length of $\sim 200 \mathrm{~km}$ at midlatitudes, finer subdivision may be justified: the LES results shown here, with structures of a few hundred metres in length abundant within the domain, indicate that dust lifting hotspots could develop on scales of as little as $0.001 \%$ of the MGCM gridbox area.

To parameterise gustiness in a GCM, in both a spatial and a temporal sense, a single gridbox value of wind speed or drag velocity can be replaced by a probability density function (pdf), representing the range of values occurring within the gridbox and over the length of the timestep. A very commonly used function for modelling wind variability is the Weibull distribution [e.g., Justus et al., 1978, Gillette and Passi, 1988], with scale parameter equal to the ambient drag velocity or wind speed: Fenton and Michaels [2010] found that this distribution, with a shape parameter $k$ of $2.5-3.0$ and scale parameter $u_{*}$, provided an adequate description of the drag velocities produced by their Martian LES model (however, they noted that wind speeds deviated from the Weibull distribution when strong ambient winds were present). A similar Weibull-like distribution is obtained using our LES model, an example of which is shown in the histogram and overlaid curve of Fig. 8.

Efforts have already been made to parameterise winds using Weibull distributions in a mesoscale model, by Michaels [2006], and in an MGCM, by Newman et al. [2002b], though in the latter case a too-small value of $k$ (based on data available at the time) was used, resulting in widespread dust lifting and underestimated interannual variability. Here we aim to utilise the more realistic $k$ values provided by Fenton and Michaels [2010], and additionally to include a plausible limit on the impact of windspeed variability on dust emission (sug- 
gested by Newman et al. [2002b] as a possible improvement to their method), through the method outlined in the next paragraph.

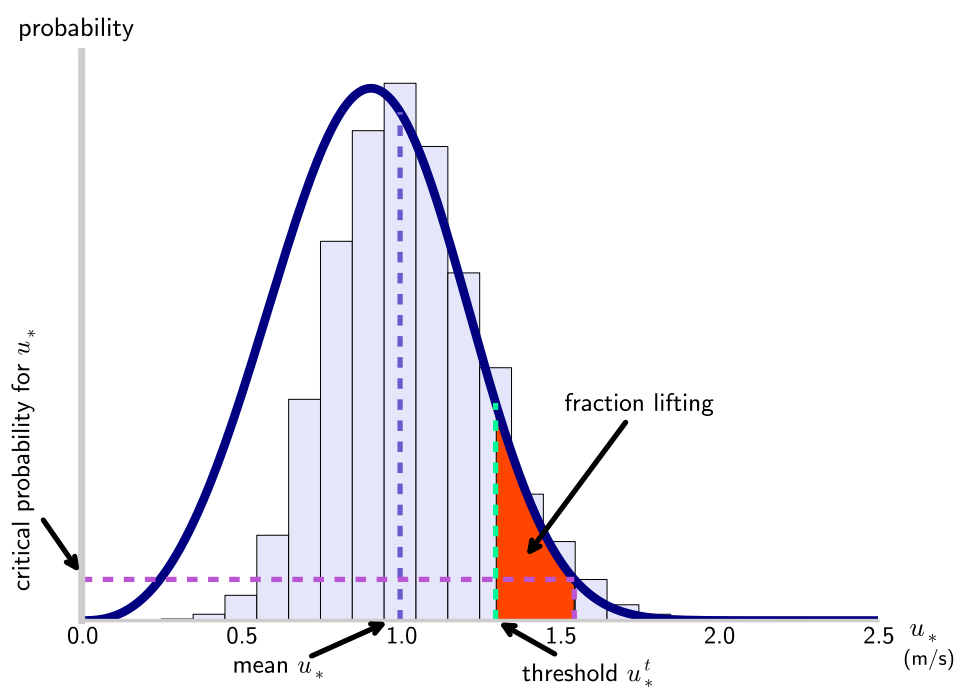

Fig. 8. Frequency of occurrence of $u_{* \text { sub }}$ values in a simulation with the LMD LES model (taking values from throughout the domain during the period of peak convection in early afternoon) using an ambient $u_{*}$ of $1 \mathrm{~m} \mathrm{~s}^{-1}$ (histogram). Overlaid is a Weibull pdf with a scale parameter of $1 \mathrm{~m} \mathrm{~s}^{-1}$ and a shape parameter of $k=3.5$ (blue curve). An illustrative threshold drag velocity $u_{*}^{t}$ is also shown (green dashed line) to highlight the dust lifting that can occur (red shaded area) when mean $u_{*}$ is below the threshold, if a probability distribution (with a certain critical probability) is used for $u_{*, \text { sub }}$.

Accounting for sub-grid scale variability in surface wind allows dust lifting activation in areas with larger mean thresholds than would otherwise be the case. The limit to this increase is set by the cut-off point applied to the tail of the pdf (which is where lifting hotspots are found), to represent a critical (minimum) probability, in space and/or time, with which a particular wind speed must occur in order to be considered as a possible initiator of a dust lifting event. The physical intuition behind this is that there exists some limit 
on the size of departures from the mean wind speed that can occur within a finite area and time, as Weibull-like wind distributions cannot be applied indefinitely down to arbitrarily small scales in space and/or time. Since dust lifting has a strongly non-linear dependence on wind speed, it will always be the case that extremely low-probability gust events from the tail of the pdf produce some amount of dust flux in a gridbox, even if the mean wind speed lies far below the threshold, unless a critical probability is defined. Additionally, when very short or spatially limited bursts of saltation do occur, the mobilised dust may in reality be unlikely to become entrained into and mixed throughout the PBL (whereas in the MGCM, this occurs rapidly once dust is injected into the lowest model level). The use of a critical probability also ensures that the sub-grid scale parameterisation is applied only on scales at which it is physically consistent, and the introduction of this parameter allows us to quantify the increasing impact of sub-grid scale variability as smaller and smaller scales are accounted for.

As an example, a probability (or frequency) of 0.01 can be interpreted equivalently as a gust lasting one-hundredth of a timestep ( $\sim 30$ seconds), covering $1 \%$ of the gridbox area $\left(\sim 400 \mathrm{~km}^{2}\right)$, or a combination of the two (from ergodic theory, which says that the behaviour of an ensemble of identical systems is 615 equivalent to the behaviour of a single realisation over a longer time period, spatial and temporal variability can be used interchangeably in this context). Hence, Fig. 7 suggests that the critical probability could be at least as low as $10^{-5}$ (structures are seen down to the model resolution of $100 \mathrm{~m}$ ). The situation is illustrated in Fig. 8: for a particular threshold $u_{*}^{t}$, dust lifting can occur 620 with an ambient $u_{*}<u_{*}^{t}$ if the gustiness is strong enough and the critical probability is sufficiently low. 


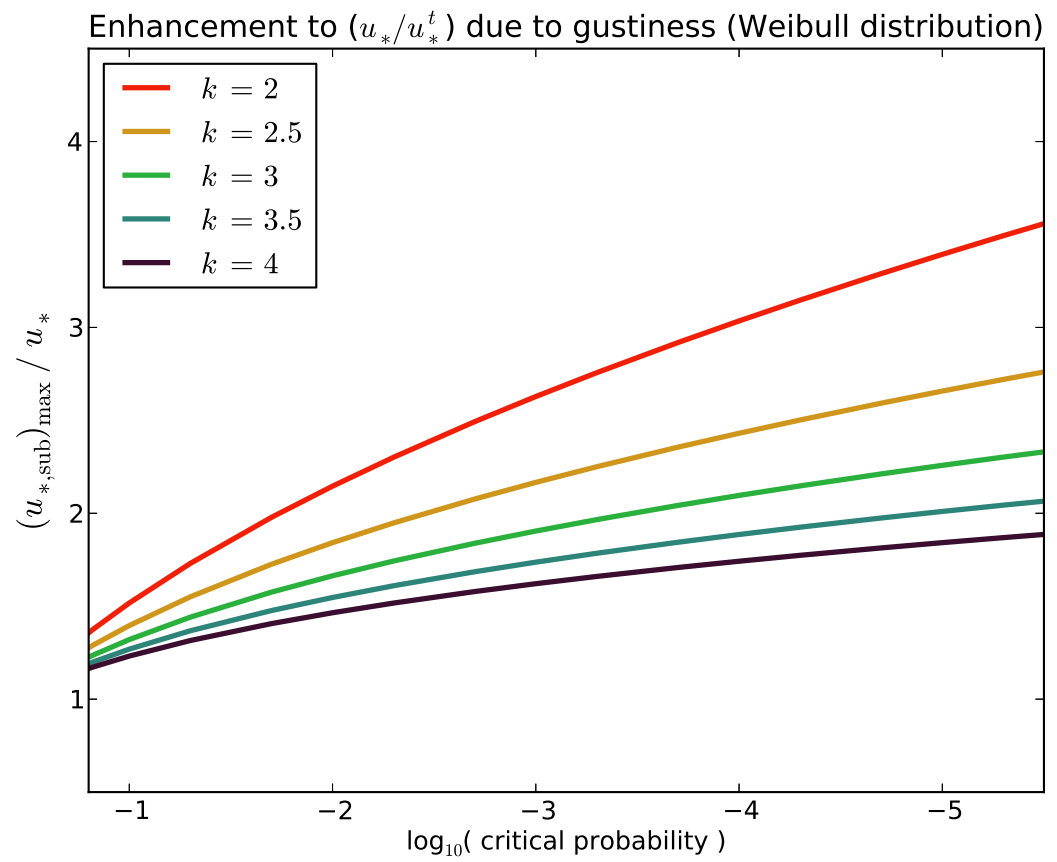

Fig. 9. The ratio $\frac{\left(u_{*, \mathrm{sub}} / u_{*, \mathrm{sub}}^{t}\right)_{\max }}{u_{*} / u_{*}^{t}}$, accounting for sub-grid scale wind speed variability (for a constant $z_{0}$ ) using a Weibull distribution with a range of $k$ values, as a function of critical probability (note that this is equal to $\left.\frac{\left(u_{*, \mathrm{sub}}\right)_{\max }}{u_{*}}\right)$. Critical probabilities as low as $10^{-5}$ are suggested by Fig. 7 .

Fig. 9 shows the relative increase to $u_{*} / u_{*}^{t}$ caused by gustiness, modelled with Weibull distributions with various shape parameters. Since thresholds are constant, this is equal to $\left(u_{*, \mathrm{sub}}\right)_{\max } / u_{*}$. It is clear that substantial enhancements to a model's ability to lift dust will result, even with a relatively low cut-off point for the drag velocity pdf. With $k=2.5-3.0$ and a critical probability $<10^{-3}$, peak sub-grid winds $\left(u_{*, \text { sub }}\right)_{\max }$ are $2-3$ times as strong as the mean wind $u_{*}$. There is agreement here with Fig. 7 too: the LES simulation produced peaks (at each gridpoint) of 1.5-2.5 times the mean $u_{*}$ when run for one sol with a timestep of 1.5 seconds, equivalent to a critical probability of $\mathrm{O}\left(10^{-5}\right)$, which is in line with Fig. 9, for $k$ values of $\sim 2.5-4$. 
The values here represent the scaling factor that may be applied to the $u_{*} / u_{*}^{t}$ field shown in Fig. 4, to account for unresolved turbulent gustiness, and may be compared to those in Fig. 6, which shows the same for unresolved $z_{0}$ variability, with a critical probability of 0.001 (representing the change in spatial resolution from $5^{\circ}$ to $\sim \frac{1}{6}^{\circ}$ ). If a globally homogeneous $k=3.0$ is assumed, $u_{*} / u_{*}^{t}$ is increased, due to gustiness, by around a factor of two at all gridpoints, meaning that in the southern midlatitudes, variability in $z_{0}$ and in wind speed could have comparable impacts on dust lifting.

Note that although the Weibull distribution accounts for the full range of wind speed variability within an MGCM gridbox, it cannot fully account for mesoscale structure in the wind field, in particular from slope winds, which are caused by heterogeneity in the surface topography that is seen by neither the MGCM nor the sub-grid scale parameterisation. We return to this point in section 5 , but stress that a gustiness parameterisation method of this kind, with a globally homogeneous shape parameter $k$, does not provide the complete solution to the problem of unresolved wind variability in an MGCM.

\section{Combined impact of sub-grid variabilities on dust lifting}

Taken together, the changes to $u_{*} / u_{*}^{t}$ resulting from the parameterisation of sub-grid scale variability in $z_{0}$ and in surface wind speed are substantial, and imply that dust lifting rates are very different, both in magnitude and in spatial distribution, than those which are produced by calculation at MGCM spatial resolution. We now explore quantitatively the possible impact of these sub-grid scale variabilities, by returning to the MCD data introduced in section 2 . 
Sub-grid scale variability is modelled stochastically, by drawing random numbers to construct pdfs for each of $z_{0}$ and near-surface wind speed. For surface roughness, values are distributed log-normally about the gridbox mean, using either a prescribed, spatially uniform geometric standard deviation $\sigma_{g}\left(z_{0}\right)$ (ranging from 1.0, resulting in no variability, to 2.0), or a heterogeneous $\sigma_{g}\left(z_{0}\right)$ calculated from the high-resolution $z_{0}$ map (as in section 3.1; see also Supplementary Information, Fig. S2). For gustiness, a Weibull distribution is used for $u\left(z_{1}\right)_{\text {sub }}$ (rather than $u_{* \text {,sub }}$ which, due to the heterogeneous $z_{0}$ used in conversion from $u\left(z_{1}\right)_{\text {sub }}$, will now in general not follow a Weibull distribution), with the ambient wind speed $u\left(z_{1}\right)$ as the scale parameter and a spatially homogeneous shape parameter $k$ in the range 2.5-3.5, and these wind speeds are then converted to drag velocities $u_{* \text {,sub }}$ using the heterogeneous $z_{0}$ values from the generated pdf. Finally, for both variables, a critical probability is chosen to define the cut-off point for the tails of the distributions. Physically, if expressed as a fraction of the gridbox area, this parameter represents the smallest scale of variability that is considered in calculation of the lifted dust flux for the gridbox. Using this approach, arrays of $u_{*, \text { sub }}$ and $u_{* \text { sub }}^{t}$ are calculated in each gridbox for each 30-minute timestep in a typical model year. Further details on these calculations are given in the Supplementary Information.

Saltation fluxes can then be calculated, using the common saltation flux $(H)$ formula from White [1979], which has

$$
H\left(u_{*}, u_{*}^{t}\right) \propto \frac{\rho}{g}\left(u_{*}^{t}\right)^{3}\left(\frac{u_{*}}{u_{*}^{t}}-1\right)\left(\frac{u_{*}}{u_{*}^{t}}+1\right)^{2} .
$$

Fluxes with and without sub-grid scale variability are therefore, respectively,

$$
H_{\text {sub }}=\frac{1}{N} \sum_{i=1}^{N} H\left(u_{*, \mathrm{sub}}, u_{*, \mathrm{sub}}^{t}\right)_{i} \quad \text { and } \quad H_{\text {mean }}=H\left(u_{*}, u_{*}^{t}\right)
$$


where $N$ is the number of sub-grid boxes being used. Dust lifting rate (ver-

tical flux) is generally taken to be proportional to $H$, with a proportionality constant, the 'lifting efficiency', that is dependent on the particle size distribution present at the surface, as well as other factors, and the value of which is extremely poorly known for Mars (more complex schemes for the relationship between horizontal and vertical flux exist, but exhibit very large uncertainty at present [Kang et al., 2011]). Saltation fluxes, when shown in this section, are normalised, and as a result can equivalently be interpreted as normalised dust lifting rates.

With both gustiness and $z_{0}$ variability included, the situation shown in Fig. 8 must now be updated to include a spatially varying lifting threshold. The interaction of the two pdfs, of $u\left(z_{1}\right)_{\text {sub }}$ and $u\left(z_{1}\right)_{\text {sub }}^{t}$, is illustrated in the diagram in Fig. 10, in which the orange shaded area represents a collection of dust lifting hotspots within the gridbox. Also shown in Fig. 10 is an example of the stochastic sampling approach, where lifting at several hotspots can be seen for a sub-threshold mean wind speed. At this hypothetical gridpoint, mean $z_{0}$ is $0.5 \mathrm{~cm}$ with $\sigma_{g}\left(z_{0}\right)=1.5$, and sub-grid wind speeds follow a Weibull distribution with $k=2.5$. The mean wind speed at $z_{1}$ is set to half the threshold value. Despite this, dust lifting occurs at multiple sites within the gridbox: primarily those at which strong gusts occur, with some weighting towards low values of $z_{0}$.

The sensitivity of dust lifting rates to the parameters $k$ and $\sigma_{g}\left(z_{0}\right)$, which control the magnitudes of each of the two types of variability, is explored below; first, results from the most plausible pair of parameters - a gustiness shape parameter of $k=3.0$ and $\sigma_{g}\left(z_{0}\right)$ calculated from the high-resolution $z_{0}$ map - are shown. Fig. 11 shows peak $u_{*, \text { sub }} / u_{* \text { sub }}^{t}$ resulting from the inclusion of 

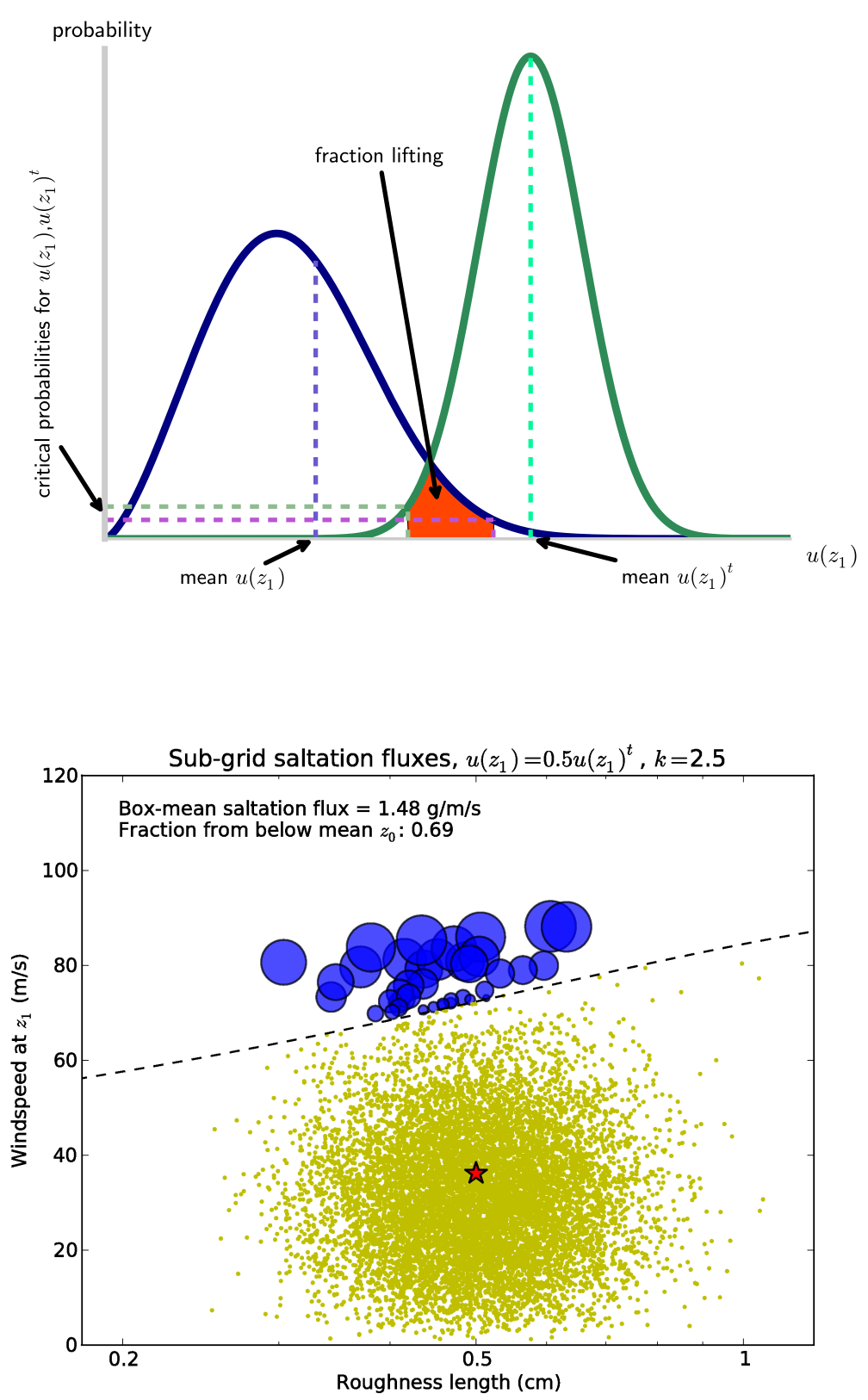

Fig. 10. (Top) Diagram (an extension of Fig. 8, with Weibull distributions used for wind speed rather than drag velocity) illustrating how pdfs for $u\left(z_{1}\right)_{\text {sub }}$ (blue) and $u\left(z_{1}\right)_{\text {sub }}^{t}$ (green) can interact to produce dust lifting in a portion of the gridbox (note that the $u\left(z_{1}\right)_{\text {sub }}^{t}$ distribution is in reality more complex than the normal distribution drawn here). (Bottom) Saltation fluxes (from (6)) at 10,000 points (i.e. assuming a critical probability of 0.0001$)$ sampled in the $\left(u\left(z_{1}\right)_{\mathrm{sub}}, z_{0, \mathrm{sub}}\right)$ domain, within a gridbox with $z_{0}=0.5 \mathrm{~cm}, \sigma_{g}\left(z_{0}\right)=1.5, k=2.5$ and $u\left(z_{1}\right)=0.5 u\left(z_{1}\right)^{t}$. The red star marks the mean values of wind speed and roughness length. The dashed line marks the threshold for lifting, $u\left(z_{1}\right)_{\mathrm{sub}}^{t}$. Points at which dust lifting occurs are plotted as blue circles, with sizes proportional to the local dust flux; points at which no lifting occurs are plotted as yellow dots. 

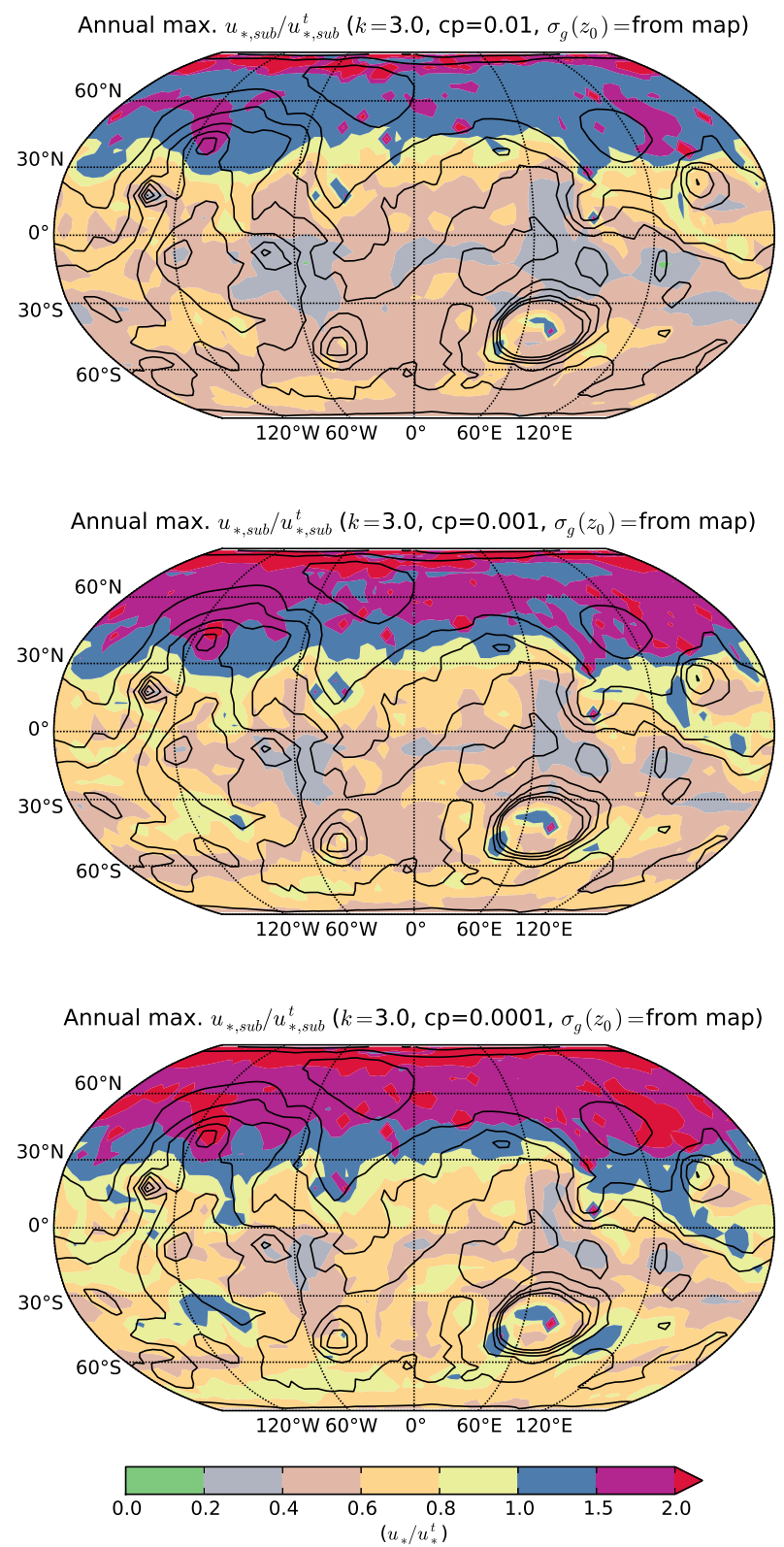

Fig. 11. Annual maximum $u_{*, \text { sub }} / u_{* \text { sub }}^{t}$ from the MCD, as in Fig. 4 , when sampling sub-grid scale variability in $z_{0}$, using geometric standard deviations taken from the high-resolution map, and in wind speed, using a Weibull distribution with shape parameter $k=3.0$ and scale parameter $u\left(z_{1}\right)$. Critical probabilities of 0.01 (top), 0.001 (middle) and 0.0001 (bottom) are shown. 
both types of sub-grid scale variability, where now the value for a gridbox is the maximum of any of the sub-grid areas within that gridbox. It is seen that even with a relatively low critical probability of 0.01 , substantial increases over the mean-fields case, shown in Fig. 4, are produced: the combined impacts of the two types of variability cause the annual maximum $u_{*, \mathrm{sub}} / u_{* \text {,sub }}^{t}$ to exceed unity throughout the northern mid- and high-latitudes, and dust lifting also becomes possible, at least briefly, at a greater number of points in the southern hemisphere, on the western and northeastern slopes of Hellas. With a critical probability of 0.001 , further southern midlatitude dust lifting sites are activated, at Solis Planum and east of Hellas. Furthermore, large areas of the southern hemisphere now show peak drag velocities not far below the local threshold. It is possible that some of these areas may become viable for dust lifting during a major dust storm, when surface stresses are elevated due to the strengthened global circulation [Haberle et al., 1982, Montabone et al., 2005]. The activation of secondary lifting sites may in fact be a crucial part of the development of some major dust storms [Strausberg et al., 2005]. With a critical probability of 0.0001 - still very plausible, at least with respect to gustiness, according to Fig. 7 - further southern hemisphere areas reach or come close to reaching the threshold at some point during the year, including the south polar region, where cap-edge dust storms have been observed [e.g., Imamura and Ito, 2011]. The percentages of gridpoints at which the threshold is reached in the three cases described are 30\%,34\% and 38\% respectively.

Similar plots for separate inclusions of each effect (with critical probability 0.001) are provided in Supplementary Information, Fig. S4. For these parameter choices, the impacts of the two forms of variability are comparable in some locations, but gustiness has the bigger impact overall (12\% of gridpoints 
exceed the threshold in the $z_{0}$-only case, while $29 \%$ do so in the gustinessonly case, compared to $4 \%$ for the mean fields case (Fig. 4)). Increases in peak $u_{* \text {,sub }} / u_{* \text {,sub }}^{t}$ (compared to $\left.u_{*} / u_{*}^{t}\right)$ due to gustiness occur globally homogeneously, since a uniform $k$ is used, whereas increases due to $z_{0}$ variability are greatest at $30-60^{\circ} \mathrm{S}$ and virtually absent in the plains at $0-20^{\circ} \mathrm{N}$ (compare with Fig. 4), due to the sensitivity (insensitivity) of the lifting threshold to $z_{0}$ in rough (smooth) regions. Roughness length variability increases in importance, relative to gustiness, as the critical probability is decreased (see also Fig. 12), as additional southern hemisphere gridpoints become activated via substantially lowered thresholds. The relative importance of the two variabilities ultimately depends on the critical probabilities, which need not necessarily be the same for each in reality.

The sensitivity of global dust lifting rates to the various sub-grid scale parameters used is shown in Fig. 12. Total lifting rates increase with decreasing $k$ and increasing $\sigma_{g}\left(z_{0}\right)$. Gustiness dominates in these results, though this could be partly due to the handling of $z_{0, \text { sub }}$ at large mean roughness lengths $z_{0, \text { sub }}$ values greater than $2 \mathrm{~cm}$ can be generated by the sampling method, but no lifting is allowed to occur from these sub-grid points, in order not to move beyond the applicable range of the $f_{\text {eff }}$ function used (and with the expectation that lifting rates ultimately become negligible at large $z_{0}$ ). If instead an upper limit is placed on the $z_{0 \text {,sub }}$ values that can be stochastically generated, resulting global mean lifting rates increase more substantially with increasing $\sigma_{g}\left(z_{0}\right)$, but the variation due to gustiness remains the larger of the two sensitivities, for the parameter ranges considered.

The sensitivity to both types of sub-grid variability increases as the critical probability is reduced, as the tails of the generated pdfs become more thor- 


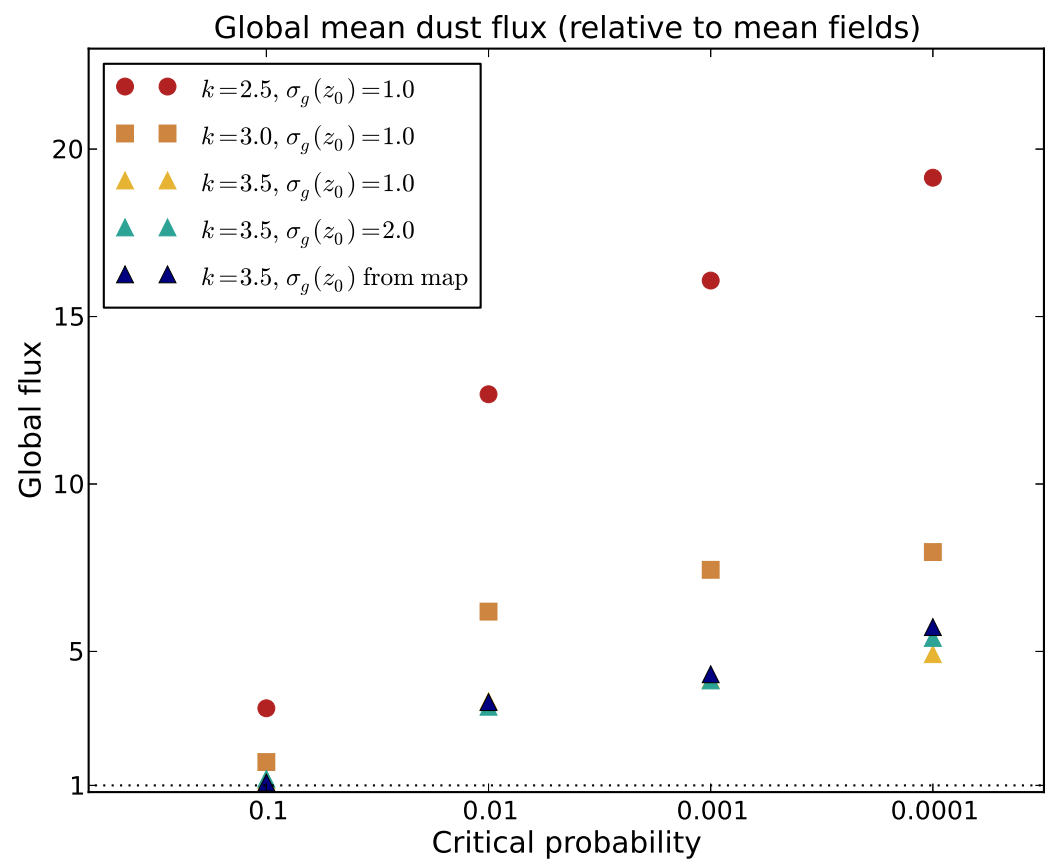

Fig. 12. A summary of global mean dust fluxes (lifting rates) for various combinations of sub-grid variability parameters, allowing lifting only at ice-free gridpoints, with roughness lengths no larger than $2 \mathrm{~cm}$. Lifting rates are expressed relative to the value obtained using mean fields; i.e. $H_{\text {sub }} / H_{\text {mean }}$ is plotted, with the mean field baseline $H_{\text {mean }}$ represented by the dotted line at a value of 1 .

oughly sampled. Lifting rates increase with decreasing critical probability, as does the sensitivity to $\sigma_{g}\left(z_{0}\right)$, as greater numbers of low- $z_{0}$ sub-grid points (with low $u_{*}^{t}$ ) are sampled. Increases to total dust lifting rates are substantial, for most plausible choices of parameters, even for relatively high critical probabilities.

Fig. 13 shows the global distribution of annual mean dust lifting, in the form of the saltation flux normalised by the largest value on the planet. With $k=3.0$, a critical probability of 0.001 and $z_{0}$ variability based on the high-resolution map, the figure shows that dust lifting is predicted to occur most frequently in 
the northern midlatitudes, particularly around Acidalia Planitia $\left(\sim 90-0^{\circ} \mathrm{W}\right.$, $50-70^{\circ} \mathrm{N}$ ), from where flushing storms have been seen to originate most regularly [e.g., Hinson et al., 2012, Wang et al., 2013]. In fact, near Alba Patera, the threshold is exceeded on as many as half of the days of the year, suggesting a near-constant supply of atmospheric dust in this region during parts of the year - provided, that is, that dust remains available at the surface. Lifting rarely occurs, by contrast, in the low-thermal-inertia regions at $0-20^{\circ} \mathrm{N}$, which Ruff and Christensen [2002] found to possess a relatively thick surface dust layer. This supports an interpretation of these areas as present-day net dust sinks, from which emission is unlikely. The majority of wind stress dust lifting occurs in midlatitudes (around $90 \%$ of the total occurs between $35^{\circ}$ and $55^{\circ}$ ), for any level of $z_{0}$ variability assumed, as shown further in Supplementary Information, Fig. S5, where it is also seen that although increasing $\sigma_{g}\left(z_{0}\right)$ increases annual-mean lifting rates globally, rates are decreased at $15-$ $45^{\circ} \mathrm{N}$, where gridbox-mean lifting conditions are relatively favourable, as a consequence of the $1 / f_{\text {eff }}$ threshold dependence.

A number of southern midlatitude gridpoints - including those near the Hellas and Argyre basins and in other regions where dust storms have been observed [e.g., Martin, 1974, Wang et al., 2013] — are also predicted to produce dust lifting on multiple occasions during the year. Although the large thresholds in these regions do make simulated dust lifting events rarer than they are in the northern hemisphere, it should be noted that the $u_{*}{ }^{3}$ dependence in the saltation flux formula (equation (6)) means that when the threshold is exceeded at a gridpoint with large $z_{0}$ (requiring a large $u_{*}$ ), saltation tends to occur with greater intensity, and so it may be that lifting events in the southern midlatitudes have a greater chance of growing into local or regional 


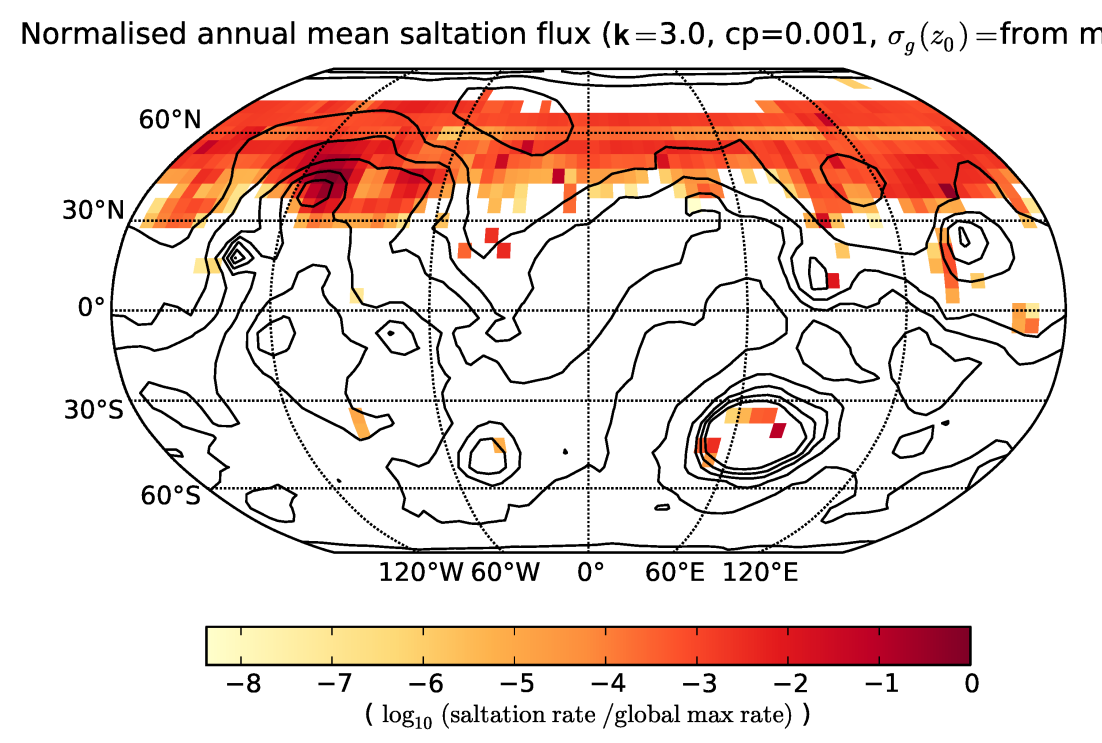

Fig. 13. Saltation flux (to which dust lifting rate is often set proportional), normalised by the largest value on the map, as calculated from the MCD Climatology scenario, using $k=3.0$, a critical probability of 0.001 and $z_{0}$ variability calculated from the high-resolution map. Saltation and dust lifting are permitted to occur only at gridpoints without surface ice cover.

dust storms than the more common but typically less intense northern hemisphere events. (This increase of dust flux with increasing wind speed can be seen in Fig. 10, and in Supplementary Information, Fig. S6.) Also, the ratio of events in the southern to northern hemispheres is seen to increase as the critical probability is decreased, due in particular to the influence of roughness length variability, which is felt more strongly in the southern hemisphere than the northern hemisphere.

The map of predicted dust emission events bears a reasonable resemblance to the map of observed storms shown in Fig. 1, at least in the northern hemisphere. A notable discrepancy is the absence in Fig. 13 of dust lifting in the 
southern mid-to-high latitudes at levels comparable to the northern equivalent latitudes. However, these are areas that can become activated by the choice of a lower critical probability, as seen in Fig. 11. Remaining underestimation of south polar dust storm frequency may result from an under-resolving of the temperature gradient at the ice cap edge, and therefore of the strength of the baroclinic and sea-breeze flows that are crucial to the generation of these storms.

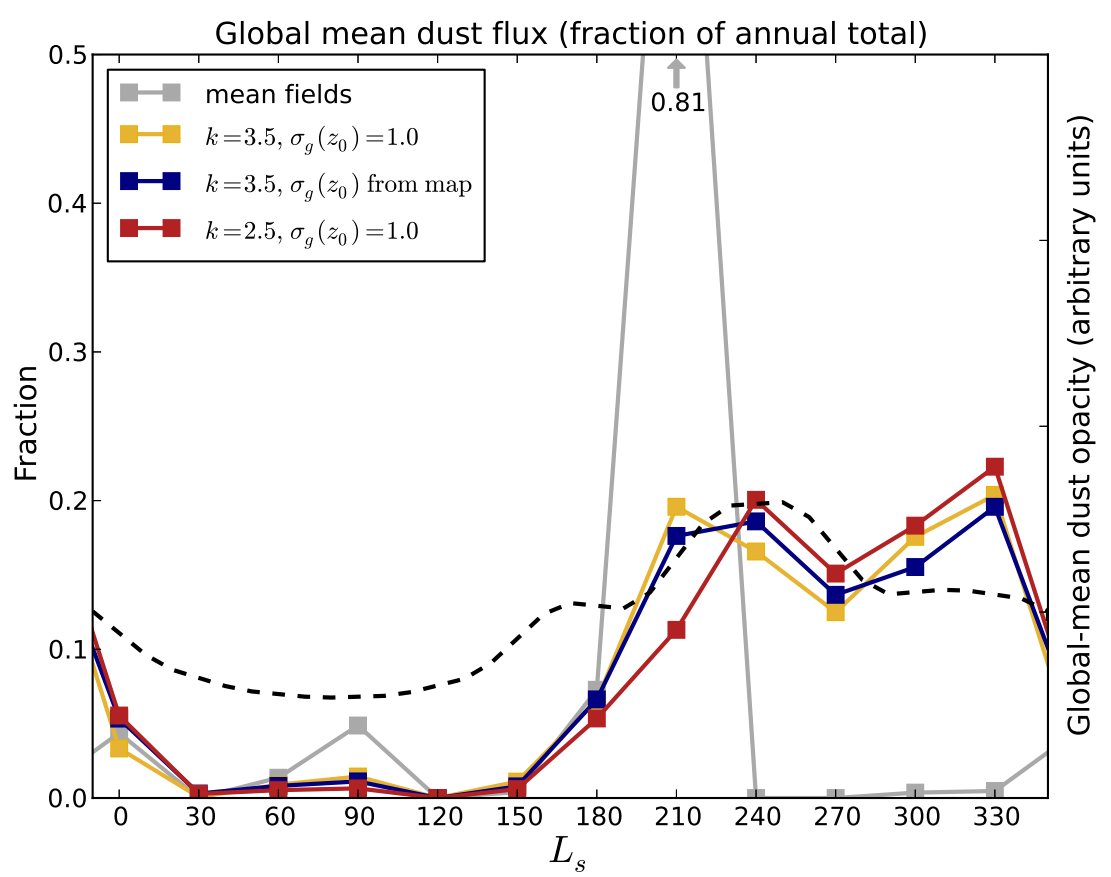

Fig. 14. Global dust lifting rates at $30^{\circ} L_{s}$ intervals, normalised by the annual totals (left axis), for mean-field (grey) and sub-grid variability (critical probability 0.001 ) cases $\left(k=2.5\right.$, red; $k=3.5$, yellow; $k=3.5$ with $z_{0}$ variability from the high-res map, blue). The dotted line shows the global-mean visible dust opacity, taken from the MCD Climatology scenario, scaled by an arbitrary factor (right axis).

Finally, the seasonal variation in dust lifting activity and its sensitivity to the various sub-grid scale parameters is shown in Fig. 14. As expected, in all cases 
the global mean vertical dust flux is largest in southern spring/summer; however, in the mean fields case (i.e. when no sub-grid scale variability is considered), the dust lifting in this part of the year is concentrated in a narrow window in southern spring. By contrast, the simulations with sub-grid variability produce lifting throughout the spring and summer seasons. A double-peaked structure is present in each of these curves over $L_{s}=180-360^{\circ}$, which is consistent with the 'solsticial pause' that has been observed to occur in flushing dust storms originating in the northern midlatitudes [Wang et al., 2005] (although the second of these peaks is not detectable in the global-mean opacity of the MCD dust scenario). Northern midlatitude storms occur with greatest frequency during $L_{s}=220-250^{\circ}$ and $L_{s}=320-330^{\circ}$ [Wang et al., 2013], and this situation is best reproduced by the strong gustiness case $(k=2.5)$.

However, it is not possible to properly discriminate between the various cases using observed dust loading time series, due to the uncertain component of this atmospheric dust that is provided by dust devils, not explicitly simulated here. The absence of dust devils is very likely the main reason for the apparent underestimation of dust lifting/loading during northern spring and summer $\left(L_{s}=30-150^{\circ}\right)$. Dust flux is increased during this period (as well as in the annual mean) with the inclusion of sub-grid variability, however, suggest825 ing that as well as small-scale 'conventional' wind stress lifting events, dust devil lifting becomes partially captured by the probabilistic representation of the high wind speeds that form in the walls of convective vortices (though a statistical wind speed distribution may not be able to fully account for convective lifting, if the efficiency of the process is enhanced by updrafts in the core of a vortex [Greeley et al., 2003]). The impact of critical probability on the seasonal cycle (not shown) appears to be small. 
To summarise, the inclusion of two forms of sub-grid scale variability in calculations of dust lifting rates increase global mean dust lifting rates substantially — by an order of magnitude, for plausible parameter values - and increase

\section{Discussion}

As Fig. 12 shows, large increases in global dust fluxes over the mean-field case are still present in this scenario, due to the sub-grid scale gustiness that remains, and in fact lifting at more than one site in the southern hemisphere is possible without sub-grid $z_{0}$ variability for a critical probability as large

Several areas of uncertainty remain in the analysis presented here. Firstly, the range of curves presented in Fig. 2 indicates that a degree of uncertainty exists over the variation of the drag partition function with $z_{0}$. While there is good reason to adopt the Raupach et al. [1993] form, the change in lifting thresholds at $z_{0} \sim 1 \mathrm{~cm}$ that would result if $f_{\text {eff }}$ were altered by only $\sim 0.1$ could have a significant impact on southern hemisphere dust lifting rates. From the point of view of parameterised MGCM dust lifting, the Raupach/Wooding form as used throughout this paper represents a dependence of lifting rates on $z_{0}$ that is large compared to other possible $f_{\text {eff }}$ forms. As a result, the correction of errors in this form seems likely to shift results in the direction of those obtained with $\sigma_{g}\left(z_{0}\right)=1$, in which $z_{0}$ sub-grid variability had no effect on lifting rates. case are still present in this scenario, due to the sub-grid scale gustiness that as 0.001 (Supplementary Information, Fig. S4). Less variable $f_{\text {eff }}$ functions 
cannot be ruled out on the basis of model lifting rates at present, since $k$ and critical probability are only loosely constrained.

Secondly, the true sub-grid scale variability of $z_{0}$ is not yet known with certainty, due in part to possible satellite artefacts present in the map presented above. It has been seen that lifting rates in the northern midlatitudes can vary by a factor of two depending on the values for $\sigma_{g}\left(z_{0}\right)$ assumed in this region, and southern midlatitudes could likewise see higher lifting rates if larger $\sigma_{g}\left(z_{0}\right)$ are present. Also, the true variability in roughness length below the resolution of the map $\left(\frac{1}{8}^{\circ}\right)$ and its possible impact on dust lifting on larger scales are unknown at present.

Some uncertainty remains over the value of $u_{* s}^{t}$, due to the possibility that direct detachment of low-density dust aggregates makes a significant or even dominant contribution to dust fluxes. However, a reduction to $u_{* s}^{t}$ of around $30 \%$ (as could result if direct detachment were in fact the mechanism responsible for wind stress dust lifting) does not dramatically change the results presented here, as the reduction applies uniformly over the planet (as far as is known currently, at least), and lifting remains extremely rare in the southern hemisphere without sub-grid scale parameterisations (see Fig. 4).

Also, a further limitation of the method, as noted earlier, is that slope winds and other mesoscale circulations are unaccounted for, except in a globally averaged sense through the Weibull distribution with fixed shape parameter. Both the slope winds themselves and their possible modification of boundary layer turbulence are important processes for dust lifting [Rafkin and Michaels, 2003], but it remains to be determined how, or even if, mesoscale topographic variation can be adequately represented in a lower-resolution global model. 


\section{Conclusions}

We have shown that when aerodynamic roughness lengths are included in the calculation of the threshold for wind stress dust lifting, parameterisation of sub-grid scale variability becomes essential to simulate lifting realistically in an MGCM. If the drag partition function of Raupach et al. [1993] is used, dust lifting is particularly impaired in the southern midlatitudes, despite this being a well-known source of dust storms of all sizes, from local to global. This shortfall in MGCM wind speeds occurs even with the use of a new surface layer calculation that accounts for varying stability conditions.

However, with parameterised sub-grid scale variability, model dust lifting rates can be increased dramatically over the values obtained when using large-scale wind and $z_{0}$ fields. A combination of mesoscale and/or turbulent variability in $z_{0}$ and near-surface gustiness - each of which contribute significantly — leads to the formation of emission hotspots: small areas in which the majority of dust lifting activity tends to occur. Capturing the spatial distribution and frequency of occurrence of these hotspots will very likely be crucial for an accurate simulation of Martian dust storms originating in the southern hemisphere, where the effect of sub-grid $z_{0}$ variability is felt particularly strongly. Lifting rates are enhanced significantly throughout the year, meaning that sub-grid scale emission could play an important role in maintaining the background low-opacity haze as well.

The obvious next step is to implement parameterisations for these two sub-grid scale effects in the MGCM, to investigate the effect on dust lifting rates in an interactive simulation in which the important positive radiative feedback on 
lifting [Newman et al., 2002b] is active. Such methods are increasingly used in terrestrial models [e.g., Zender et al., 2003, Cakmur et al., 2004, Menut et al., 2013], but may in fact be even more crucial on Mars due to the greater temporal variability in emission from key dust sources. To avoid the need for stochastic methods as employed here, analytic forms of the probability distributions used (one of which is the well-known Weibull distribution) should be sought, and the saltation flux can then be calculated by integrating under the curves, with limits determined by the minimum probability chosen (which essentially represents the extent of the increase in spatial resolution that is simulated by the parameterisation).

In all of the calculations presented, the gustiness shape parameter $k$ has assumed a spatially uniform value, yet it has been seen that variation of this parameter within its range of possible values can strongly affect lifting rates (Fig. 12). While values of around 2.5-3.0 are likely to be appropriate for the daytime boundary layer in an average sense, gustiness undoubtedly varies both spatially and seasonally. Information on this variation can be obtained from the MCD, data for which was produced by an MGCM that includes the thermal plume model of Colaïtis et al. [2013], which provides estimation of boundary layer quantities, namely PBL height and convective updraft velocity, that are reliable proxies for turbulent gustiness. Broadly speaking, gustiness (shown, 925 in the form of convective updraft velocity, in Supplementary Information, Fig. S7) is strongest in the summer hemisphere, peaking in the subtropics. In the southern hemisphere, the summer maximum stretches right around the 15$45^{\circ}$ band, and is slightly stronger than the corresponding northern hemisphere maximum, which is somewhat confined to the low-albedo, high-topography areas of Northern Tharsis, Arabia Terra and Elysium. Gustiness should therefore 
boost MGCM dust lifting most in the southern subtropics/midlatitudes during spring and summer. This, along with the asymmetric impact of $z_{0}$ variability deriving from the $1 / f_{\text {eff }}$ dependence, helps to explain how dust lifting can occur commonly in the southern midlatitudes even in the presence of broadly large roughness lengths (the contrasting situations in the northern and southern hemispheres are highlighted in Supplementary Information, Fig. S6).

A full sub-grid scale dust lifting parameterisation should include a variable gustiness intensity [as noted by Michaels, 2006], perhaps using a $k$ parameter diagnosed from boundary layer quantities such as updraft velocity, similar to the work of Cakmur et al. [2004]. The separation of dust devil and wind stress lifting mechanisms in MGCMs may complicate matters here, as this measure of gustiness, used for wind stress lifting, comes from a parameterisation that is based on LES simulations in which dust devils are partially resolved. Care must be taken to apportion the correct amount of diagnosed gustiness to each given to better representing gustiness in conditions of strong ambient winds, when a Weibull distribution may not be appropriate [Fenton and Michaels, 2010].

Accurately predicting the wind speed at which dust lifting begins is of crucial importance for MGCMs, since — at large scale — wind stress dust lifting is subject to a strong positive radiative feedback, such that one synoptic wind event in excess of the threshold can initiate an ultimately substantial dust storm. Capturing the activation point for lifting becomes even more important when considering the hysteresis idea of Kok [2010], which implies that only a brief excursion of drag velocity beyond the local threshold is needed to maintain saltation and lifting over a significant period of time, so long as the 
wind remains above a certain lower threshold, estimated to be as little as $10 \%$ of the original activation velocity. There is now observational evidence for the occurrence of this hysteresis in sand mobility on Mars [Yizhaq et al., 2014]. In this context, the LES result seen in Fig. 7 may describe a domain that, integrated over the course of day, is a very active source of dust, even if the ambient wind speed is significantly below the lifting threshold. The peak $u_{*}$ values shown typically occur at some point during the afternoon, and the hysteresis effect then suggests that saltation is likely to continue at each of those (numerous) sites throughout the afternoon and into the evening, until the boundary layer stabilises and near-surface winds weaken dramatically. The saltation hysteresis can theoretically apply on a very small spatial scale (Dupont et al. [2013] recently simulated saltation structures a few metres in length), potentially resulting in a steady supply of dust to the atmosphere from several hotspots within a given area, provided that surface dust continues to be available at those sites.

What is less clear is whether or not localised emission such as this is capable of triggering emission from neighbouring areas, via a smaller-scale positive radiative feedback than the one mentioned above [Rafkin, 2009, Spiga et al., 2013], or whether the negative feedback typical of dust devil lifting - in which radiative heating of suspended dust warms and stabilises the boundary layer, inhibiting further lifting - takes precedence at this scale. In any case, without an explicit parameterisation of this feedback (positive or negative), dust storm expansion will apply only at and above the GCM gridbox scale. Even if dust lifting from hotspots does not routinely lead to the growth of dust storms larger than a GCM gridbox, it may be the case that smaller-scale emissions are vital — probably in combination with lifting by dust devils — to 
maintaining the background dust haze. MGCMs have in general relied upon parameterised dust devil lifting to provide this dust loading, as modelled wind but the use of a sub-grid scale parameterisation has the potential to enable low levels of lifting in places from which no lifting previously occurred, increasing the background dust opacity, while retaining the potential for - under the right conditions - explosive dust storm growth.

\section{Acknowledgements}

The authors thank Benjamin Lemonnier, whose internship work provided useful insights into the global-scale dust lifting issues addressed in section 2. We thank Scot Rafkin and an anonymous reviewer for their helpful and constructive comments, which have improved this paper.

\section{References}

S. C. Alfaro and L. Gomes. Improving the large-scale modeling of the saltation flux of soil particles in presence of nonerodible elements. J. Geophys. Res., 100:16357-16366, 1995. doi: 10.1029/95JD01281.

S. P. S. Arya. A drag partition theory for determining the large-scale roughness parameter and wind stress on the arctic pack ice. J. Geophys. Res., 80(C24): 3447-3454, 1975.

R. A. Bagnold. The Physics of Blown Sand and Desert Dunes. Methuen, 1954. 
M. Balme and R. Greeley. Dust devils on Earth and Mars. Rev Geophys, 44: 1005 RG3003, September 2006. doi: 10.1029/2005RG000188.

R. V. Cakmur, R. L. Miller, and O. Torres. Incorporating the effect of smallscale circulations upon dust emission in an atmospheric general circulation model. J. Geophys. Res. (Atmospheres), 109:D07201, April 2004. doi: 10.1029/2003JD004067.

Y. Callot, B. Marticorena, and G. Bergametti. Geomorphologic approach for modelling the surface features of arid environments in a model of dust emissions: application to the sahara desert. Geodin. Acta, 13(5):245-270, 2000.

B. A. Cantor, P. B. James, M. Caplinger, and M. J. Wolff. Martian dust storms: 1999 Mars Orbiter Camera observations. J. Geophys. Res., 106: 23653-23688, oct 2001. doi: 10.1029/2000JE001310.

B. A. Cantor, K. M. Kanak, and K. S. Edgett. Mars Orbiter Camera observations of Martian dust devils and their tracks (September 1997 to January 2006) and evaluation of theoretical vortex models. J. Geophys. Res. (Planets), 111:E12002, December 2006. doi: 10.1029/2006JE002700.

A. Colaïtis, A. Spiga, F. Hourdin, C. Rio, F. Forget, and E. Millour. A thermal plume model for the martian convective boundary layer. J. Geophys. Res., 118(7):1468-1487, 2013. ISSN 2169-9100. doi: 10.1002/jgre.20104.

S. Dupont, G. Bergametti, B. Marticorena, and S. Simoëns. Modeling saltation intermittency. J. Geophys. Res., 118:7109-7128, July 2013. doi: $10.1002 /$ jgrd.50528.

S. Engelstaedter and R. Washington. Atmospheric controls on the annual cycle of north african dust. J Geophys Res, 112(D3):n/a-n/a, 2007. ISSN 2156-2202. doi: 10.1029/2006JD007195.

L. K. Fenton and T. I. Michaels. Characterizing the sensitivity of daytime 
turbulent activity on Mars with the MRAMS LES: Early results. International Journal of Mars Science and Exploration, 5:159-171, 2010. doi: 10.1555/mars.2010.0007.

J. R. Garratt. The atmospheric boundary layer. Cambridge Univ. Press, 1992.

D. A. Gillette. A qualitative geophysical explanation for 'hot spot' dust emitting source regions. Contr. Atmos. Phys., 72(1):67 - 77, 1999.

D. A. Gillette and R. Passi. Modeling dust emission caused by wind erosion. J. Geophys. Res., 93(D11):14233-14242, 1988. ISSN 2156-2202. doi: 10.1029/JD093iD11p14233.

J. Gillies, W. Nickling, and J. King. Shear stress partitioning in large patches of roughness in the atmospheric inertial sublayer. Boundary-Layer Meteorology, 122:367-396, 2007. ISSN 0006-8314. 10.1007/s10546-006-9101-5.

R. Greeley and J. D. Iversen. Wind as a geological process on Earth, Mars, Venus and Titan. Cambridge Univ. Press, 1985.

R. Greeley, M. R. Balme, J. D. Iversen, S. Metzger, R. Mickelson, J. Phoreman, and B. White. Martian dust devils: Laboratory simulations of particle threshold. J. Geophys. Res., 108(E5), 2003.

R. Greeley, P. L. Whelley, L. D. V. Neakrase, R. E. Arvidson, N. T. Bridges, N. A. Cabrol, P. R. Christensen, K. Di, D. J. Foley, M. P. Golombek, K. Herkenhoff, A. Knudson, R. O. Kuzmin, R. Li, T. Michaels, S. W. Squyres, R. Sullivan, and S. D. Thompson. Columbia Hills, Mars: Aeolian features seen from the ground and orbit. J. Geophys. Res. (Planets), 113:E06S06, April 2008. doi: 10.1029/2007JE002971.

R. M. Haberle, C. B. Leovy, and J. B. Pollack. Some effects of global dust 1055 storms on the atmospheric circulation of Mars. Icarus, 50:322-367, jun 1982. doi: 10.1016/0019-1035(82)90129-4.

N. G. Heavens, M. I. Richardson, and A. D. Toigo. Two aerodynamic rough- 
ness maps derived from mars orbiter laser altimeter (mola) data and their effects on boundary layer properties in a mars general circulation model (gcm). J. Geophys. Res., 113(E2), 2008.

E. Hébrard, C. Listowski, P. Coll, B. Marticorena, G. Bergametti, A. Määttänen, F. Montmessin, and F. Forget. An aerodynamic roughness length map derived from extended Martian rock abundance data. J. Geophys. Res. (Planets), 117:E04008, April 2012. doi: 10.1029/2011JE003942.

D. P. Hinson, H. Wang, and M. D. Smith. A multi-year survey of dynamics near the surface in the northern hemisphere of Mars: Short-period baroclinic waves and dust storms. Icarus, 219:307-320, May 2012. doi: 10.1016/j.icarus.2012.03.001.

F. Hourdin, F. Forget, and O. Talagrand. The sensitivity of the Martian sur1070 face pressure and atmospheric mass budget to various parameters: A comparison between numerical simulations and Viking observations. J. Geophys. Res., 100:5501-5523, mar 1995. doi: 10.1029/94JE03079.

T. Imamura and Y. Ito. Quasi-periodic dust events in the summertime south polar region of mars. Icarus, 211(1):498-503, 2011.

J. D. Iversen and B. R. White. Saltation threshold on earth, mars and venus. Sedimentology, 29(1):111-119, 1982.

B. M. Jakosky, B. M. Hynek, S. M. Pelkey, M. T. Mellon, S. Martínez-Alonso, N. E. Putzig, N. Murphy, and P. R. Christensen. Thermophysical properties of the mer and beagle ii landing site regions on mars. J. Geophys. Res., 111 $1080 \quad(\mathrm{E} 8), 2006$.

C. G. Justus, W. R. Hargraves, A. Mikhail, and D. Graber. Methods for estimating wind speed frequency distributions. J. Appl. Meteorol., 17(3): 350-353, 1978.

M. A. Kahre, J. R. Murphy, and R. M. Haberle. Modeling the Martian 
dust cycle and surface dust reservoirs with the NASA Ames general circulation model. J. Geophys. Res. (Planets), 111:E06008, June 2006. doi: 10.1029/2005JE002588.

J.-Y. Kang, S.-C. Yoon, Y. Shao, and S.-W. Kim. Comparison of vertical dust flux by implementing three dust emission schemes in wrf/chem. J Geophys Res, 116(D9), 2011. ISSN 2156-2202. doi: 10.1029/2010JD014649.

J. F. Kok. Analytical calculation of the minimum wind speed required to sustain wind-blown sand on Earth and Mars. ArXiv e-prints, jan 2010.

J. F. Kok, E. J. R. Parteli, T. I. Michaels, and D. B. Karam. The physics of wind-blown sand and dust. Rep Prog Phys, 75(10):106901, October 2012. doi: 10.1088/0034-4885/75/10/106901.

B. Laurent, B. Marticorena, G. Bergametti, J. F. Léon, and N. M. Mahowald. Modeling mineral dust emissions from the Sahara desert using new surface properties and soil database. J. Geophys. Res. (Atmospheres), 113:D14218, July 2008. doi: 10.1029/2007JD009484.

A. Määttänen, H. Vehkamäki, A. Lauri, S. Merikallio, J. Kauhanen, H. Savijärvi, and M. Kulmala. Nucleation studies in the Martian atmosphere. J. Geophys. Res.-Planets, 110:E02002, February 2005. doi: 10.1029/2004JE002308.

A. Määttänen, T. Fouchet, O. Forni, F. Forget, H. Savijärvi, B. Gondet, R. Melchiorri, Y. Langevin, V. Formisano, M. Giuranna, and J.-P. Bibring. A study of the properties of a local dust storm with Mars Express OMEGA and PFS data. Icarus, 201:504-516, June 2009. doi: 10.1016/j.icarus.2009.01.024.

D. J. MacKinnon, G. D. Clow, R. K. Tigges, R. L. Reynolds, and P. S. Chavez. Comparison of aerodynamically and model-derived roughness lengths (z"o) over diverse surfaces, central Mojave Desert, California, USA. Geomorphol- 
ogy, 63:103-113, November 2004. doi: 10.1016/j.geomorph.2004.03.009.

J. K. Marshall. Drag measurements in roughness arrays of varying density and distribution. Agr Meteorol, 8:269-292, 1971.

B. Marticorena and G. Bergametti. Modeling the atmospheric dust cycle: 1. Design of a soil-derived dust emission scheme. J. Geophys. Res., 100: 16415-16430, 1995. doi: 10.1029/95JD00690.

L. J. Martin. The major martian yellow storm of 1971. Icarus, 22:175-188, June 1974. doi: 10.1016/0019-1035(74)90116-X.

L. Menut, C. Pérez, K. Haustein, B. Bessagnet, C. Prigent, and S. Alfaro. Impact of surface roughness and soil texture on mineral dust emission fluxes modeling. J Geophys Res, 118(12):6505-6520, 2013. ISSN 2169-8996. doi: $10.1002 /$ jgrd.50313.

J. Merrison, J. Jensen, K. Kinch, R. Mugford, and P. Nørnberg. The electrical 1125 properties of mars analogue dust. Planet. Space Sci., 52(4):279-290, 2004.

J. P. Merrison, H. P. Gunnlaugsson, P. Nørnberg, A. E. Jensen, and K. R. Rasmussen. Determination of the wind induced detachment threshold for granular material on Mars using wind tunnel simulations. Icarus, 191:568580, November 2007. doi: 10.1016/j.icarus.2007.04.035.

T. I. Michaels. Numerical modeling of mars dust devils: Albedo track generation. Geophys. Res. Lett., 33(19), 2006.

T. I. Michaels and S. C. R. Rafkin. Large-eddy simulation of atmospheric convection on Mars. Q J Roy Meteor Soc, 130:1251-1274, April 2004. doi: 10.1256/qj.02.169.

E. Millour, A. Spiga, A. Colaïtis, T. Navarro, J.-B. Madeleine, J.-Y. Chauffray, L. Montabone, M. A. Lopez-valverde, F. Gonzalez-Galindo, F. Lefèvre, F. Montmessin, S. R. Lewis, L. P. Read, M.-C. Desjean, and J.-P. Huot. Mars Climate Database Version 5. In European Planetary Science Congress 
2012, page 302, September 2012.

AS Monin and AMf Obukhov. Basic laws of turbulent mixing in the surface layer of the atmosphere. Contrib. Geophys. Inst. Acad. Sci. USSR, 151: $163-187,1954$.

L. Montabone, S. R. Lewis, and P. L. Read. Interannual variability of Martian dust storms in assimilation of several years of Mars global surveyor observations. Adv. Sp. Res., 36:2146-2155, 2005. doi: 10.1016/j.asr.2005.07.047.

D. P. Mulholland, P. L. Read, and S. R. Lewis. Simulating the interannual variability of major dust storms on mars using variable lifting thresholds. Icarus, 223(1):344-358, 2013.

J. R. Murphy, J. B. Pollack, R. M. Haberle, C. B. Leovy, O. B. Toon, and J. Schaeffer. Three-dimensional numerical simulation of Martian global dust storms. J. Geophys. Res., 100:26357-26376, 1995. doi: 10.1029/95JE02984.

C. E. Newman, S. R. Lewis, P. L. Read, and F. Forget. Modeling the Martian dust cycle, 1. Representations of dust transport processes. J. Geophys. Res. (Planets), 107:5123-+, dec 2002a. doi: 10.1029/2002JE001910.

C. E. Newman, S. R. Lewis, P. L. Read, and F. Forget. Modeling the Martian dust cycle 2. Multiannual radiatively active dust transport simulations. J. Geophys. Res. (Planets), 107:5124-+, dec 2002b. doi: 10.1029/2002JE001920.

S. A. Nowicki and P. R. Christensen. Rock abundance on Mars from the Thermal Emission Spectrometer. J. Geophys. Res. (Planets), 112:E05007, May 2007. doi: 10.1029/2006JE002798.

G. S. Okin. Dependence of wind erosion and dust emission on surface heterogeneity: Stochastic modeling. J. Geophys. Res. (Atmospheres), 110(D9): 11208-+, jun 2005. doi: 10.1029/2004JD005288.

G. S. Okin. A new model of wind erosion in the presence of vegetation. $J$. 
Geophys. Res., 113(F12):F02S10, mar 2008. doi: 10.1029/2007JF000758.

Y.-K. Park and S. H. Park. Development of a new wind-blown-dust emission module using comparative assessment of existing dust models. Particul Sci Technol, 28(3):267-286, 2010. doi: 10.1080/02726351.2010.491761.

C. H. B. Priestley. Turbulent transfer in the lower atmosphere. University of Chicago Press, Chicago, USA, 1959.

S. C. R. Rafkin. A positive radiative-dynamic feedback mechanism for the maintenance and growth of martian dust storms. J Geophys Res, 114(E1), 2009. ISSN 2156-2202. doi: 10.1029/2008JE003217. URL http://dx.doi.org/10.1029/2008JE003217.

S. C. R. Rafkin and T. I. Michaels. Meteorological predictions for 2003 Mars Exploration Rover high-priority landing sites. J Geophys Res, 108:8091, October 2003. doi: 10.1029/2002JE002027.

M. R. Raupach, D. A. Gillette, and J. F. Leys. The effect of roughness elements on wind erosion threshold. J. Geophys. Res., 98:3023-3029, feb 1993. doi: 10.1029/92JD01922.

N. O. Rennó, M. L. Burkett, and M. P. Larkin. A Simple Thermodynamical Theory for Dust Devils. J. Atmos. Sci., 55:3244-3252, November 1998. doi: 10.1175/1520-0469(1998)055<3244:ASTTFD>2.0.CO;2.

S. W. Ruff and P. R. Christensen. Bright and dark regions on Mars: Particle size and mineralogical characteristics based on Thermal Emission Spectrometer data. J. Geophys. Res. (Planets), 107:5127-+, dec 2002. doi: 10.1029/2001JE001580.

J. T. Schofield, J. R. Barnes, D. Crisp, R. M. Haberle, S. Larsen, J. A. Mag1190 alhaes, J. R. Murphy, A. Seiff, and G. Wilson. The Mars Pathfinder Atmospheric Structure Investigation/Meteorology. Science, 278:1752, December 1997. doi: 10.1126/science.278.5344.1752. 
Y. Shao and H. Lu. A simple expression for wind erosion threshold friction velocity. J. Geophys. Res., 105:22437-22444, sep 2000. doi: 10.1029/2000JD900304.

M. D. Smith. Interannual variability in TES atmospheric observations of Mars during 1999-2003. Icarus, 167:148-165, jan 2004. doi: 10.1016/S00191035(03)00287-2.

M. D. Smith, M. J. Wolff, N. Spanovich, A. Ghosh, D. Banfield, P. R. Christensen, G. A. Landis, and S. W. Squyres. One Martian year of atmospheric observations using MER Mini-TES. J Geophys Res, 111:E12S13, December 2006. doi: 10.1029/2006JE002770.

A. Spiga and S. R. Lewis. Martian mesoscale and microscale wind variability of relevance for dust lifting. International Journal of Mars Science and Exploration, 5:146-158, 2010. doi: 10.1555/mars.2010.0006.

A. Spiga, F. Forget, S. R. Lewis, and D. P. Hinson. Structure and dynamics of the convective boundary layer on Mars as inferred from large-eddy simulations and remote-sensing measurements. Q J Roy Meteor Soc, 136:414-428, February 2010.

A. Spiga, F. Forget, J.-B. Madeleine, L. Montabone, S. R. Lewis, and E. Millour. The impact of martian mesoscale winds on surface temperature and on the determination of thermal inertia. Icarus, 212:504-519, April 2011. doi: 10.1016/j.icarus.2011.02.001.

A. Spiga, J. Faure, J.-B. Madeleine, A. Määttänen, and F. Forget. Rocket dust storms and detached dust layers in the martian atmosphere. J Geophys Res, 118(4):746-767, 2013.

M. J. Strausberg, H. Wang, M. I. Richardson, S. P. Ewald, and A. D. Toigo. Observations of the initiation and evolution of the 2001 Mars global dust storm. J. Geophys. Res. (Planets), 110(E9):2006-+, feb 2005. doi: 
R. Sullivan, R. Greeley, M. Kraft, G. Wilson, M. Golombek, K. Herkenhoff, J. Murphy, and P. Smith. Results of the Imager for Mars Pathfinder windsock experiment. J. Geophys. Res., 105:24547-24562, October 2000. doi: 10.1029/1999JE001234.

R. Sullivan, R. Arvidson, J. F. Bell, R. Gellert, M. Golombek, R. Greeley, K. Herkenhoff, J. Johnson, S. Thompson, P. Whelley, and J. Wray. Winddriven particle mobility on Mars: Insights from Mars Exploration Rover observations at "El Dorado" and surroundings at Gusev Crater. J. Geophys. Res. (Planets), 113:E06S07, June 2008. doi: 10.1029/2008JE003101.

R. Sullivan, K. Herkenhoff, J. Johnson, G. Landis, and A. Vaughan. Sand-sized dust aggregates on Mars. In Mars Dust Cycle Workshop, 2009.

J. L. Sutton, C. B. Levoy, and J. E. Tillman. Diurnal variations of the Martian surface layer meteorological parameters during the first 45 sols at two Viking Lander sites. J. Atmos. Sci., 35:2346-2355, December 1978. doi: 10.1175/1520-0469(1978)035<2346:DVOTMS>2.0.CO;2.

A. D. Toigo, M. I. Richardson, R. J. Wilson, H. Wang, and A. P. Ingersoll. A first look at dust lifting and dust storms near the south pole of Mars with a mesoscale model. J. Geophys. Res. (Planets), 107:5050, July 2002. doi: 10.1029/2001JE001592.

D. Tyler, J. R. Barnes, and R. M. Haberle. Simulation of surface meteorology at the Pathfinder and VL1 sites using a Mars mesoscale model. J Geophys Res, 107:5018, April 2002. doi: 10.1029/2001JE001618.

H. Wang, R. W. Zurek, and M. I. Richardson. Relationship between frontal dust storms and transient eddy activity in the northern hemisphere of Mars as observed by Mars Global Surveyor. J. Geophys. Res. (Planets), 110(E9): 7005-+, jul 2005. doi: 10.1029/2005JE002423. 
H. Wang, M. I. Richardson, A. D. Toigo, and C. E. Newman. Zonal wavenumber three traveling waves in the northern hemisphere of Mars simulated with a general circulation model. Icarus, 223:654-676, April 2013. doi: 10.1016/j.icarus.2013.01.004.

B. R. White. Soil transport by winds on Mars. J. Geophys. Res., 84:4643-4651, aug 1979. doi: 10.1029/JB084iB09p04643.

S. A. Wolfe and W. G. Nickling. Shear Stress Partitioning in Sparsely Vegetated Desert Canopies. Earth Surface Processes and Landforms, 21(7): 607-619, July 1996. doi: 10.1002/(SICI)1096-9837(199607)21:7<607::AIDESP660>3.3.CO;2-T.

M. J. Wolff, M. D. Smith, R. T. Clancy, N. Spanovich, B. A. Whitney, M. T. Lemmon, J. L. Bandfield, D. Banfield, A. Ghosh, G. Landis, P. R. Christensen, J. F. Bell, and S. W. Squyres. Constraints on dust aerosols from the Mars Exploration Rovers using MGS overflights and Mini-TES. J. Geophys. Res. (Planets), 111(E10):12-+, dec 2006. doi: 10.1029/2006JE002786.

R. Wooding, E. Bradley, and J. Marshall. Drag due to regular arrays of roughness elements of varying geometry. Boundary-Layer Meteorology, 5: 285-308, 1973. ISSN 0006-8314. 10.1007/BF00155238.

H. Yizhaq, J. F. Kok, and I. Katra. Basaltic sand ripples at Eagle Crater as indirect evidence for the hysteresis effect in martian saltation. Icarus, 230: 143-150, February 2014. doi: 10.1016/j.icarus.2013.08.006.

C. S. Zender, D. Newman, and O. Torres. Spatial heterogeneity in aeolian erodibility: Uniform, topographic, geomorphic, and hydrologic hypotheses. $J$ 1270 Geophys Res, 108(D17), 2003. ISSN 2156-2202. doi: 10.1029/2002JD003039. URL http://dx.doi.org/10.1029/2002JD003039.

J. R. Zimbelman. Non-active dunes in the Acheron Fossae region of Mars between the Viking and Mars Global Surveyor eras. Geophys. Res. Lett., 
27:1069-1072, April 2000. doi: 10.1029/1999GL008399.

${ }_{1275}$ R. W. Zurek and L. J. Martin. Interannual variability of planet-encircling dust storms on Mars. J. Geophys. Res., 98:3247-3259, February 1993. doi: 10.1029/92JE02936. 